\title{
Quantitative Models for Prediction of Toxic Component Concentrations in Smoke Gases from FTIR Spectra
}

\author{
Hans Pottel \\ Centexbel, Scientific and Technical Research Centre of the Belgian Textile Industry, Technologiepark 7, B-9052 Zwijnaarde, Belgium
}

In this paper an overview is given of several methods for quantitative analysis of FTIR spectra. Each method has its particular advantages and disadvantages. Therefore, depending on the gas component to be analysed in smoke gas spectra, an optimal choice of method has to be made. This choice depends on several different aspects:

- Is the spectral band of interest free of interfering components?

- Does the absorption-concentration relation show strong deviations from Beer's law?

- Are significant baseline corrections necessary?

- Does the applied model need to warn for the presence of unexpected components?

- Is it sufficient to use only a few wavenumbers or is a full-spectrum method necessary?

It will not be easy to make the appropriate choice. However, in some cases, statistics can help, in others, a good rule of thumb is to keep the choice as simple as possible. (C1996 by John Wiley \& Sons, Ltd.

\section{INTRODUCTION}

Fourier Transform infrared (FTIR) spectroscopy seems to be a powerful tool for the on-line, simultaneous, quantitative determination of the concentrations of several gas components in smoke gas spectra. ${ }^{1-6}$ Although in the past few years it has become common to use infrared spectra for the quantitative analysis of complex mixtures, prior to this, infrared spectra were used primarily as a qualitative tool and for relatively simple quantitative measurements. The complex spectral features of many multicomponent data sets often made it difficult or impossible to develop reliable infrared quantitative methods.

In this paper an overview will be given of methods that can be used for quantitative determinations of multicomponent smoke gases. The advantages and disadvantages of each method will be discussed without going into the detailed mathematical background of each method. The advantages and disadvantages of each method have to be weighed and the appropriate method chosen for each of the components of interest. Attention has to be given to the choice of suitable algorithms and the possibility of automated analysis. The decision on which method to choose is sometimes not easy. Statistics can help the user to evaluate the appropriateness of the methods for the different components. Finally, some examples will be given on gas mixture spectra, showing that in most cases several methods can lead to more or less equivalent results, and in some particular cases the accuracy of the results can be very dependent on the choice of model. This article is meant as a tutorial. The reader is expected to have some knowledge of linear and matrix algebra.

\section{METHODS}

The analysis of multicomponent spectra usually consists of two steps. First, a method is constructed, based on spectra with known concentrations of the gases of interest, called the calibration or training step. The data set used for this step is called the calibration or training set. Second, the method is validated and used to predict the unknown concentrations of the gases in the spectra contained in an independent data set. This step is called the prediction or test step. The data set used in this step is the prediction or test set.

Not all the methods will be dealt with as extensively. Classical least squares (CLS) is probably the best-known quantitative method in FTIR and will be treated very extensively, together with the very popular partial least squares (PLS) method. Also, very simple tools such as peak height and peak area, which are provided with almost all the software packages delivered by the spectrometer suppliers, are considered rather extensively, since they mostly form the basis for the construction of the calibration set for the multivariate techniques.

\section{Peak height or peak area}

Although these are two methods, they are considered together. They are simple tools in which the peak height or peak area is calculated and related to the known concentration. These tools are based on Beer's law:

$$
A(\lambda)=a(\lambda) b c
$$

where $A$ is the absorbance measured at wavenumber $\lambda$, $a$ is the absorptivity, $b$ the pathlength and $c$ the concentration. Note that Beer's law assumes a linear relation between absorbance $A$ and concentration $c$ at a fixed pathlength $b$. The peak height method reads the absorbance $A$ at one peak frequency $v$ and relates it to the concentration. To avoid accidental errors at this single wavenumber or frequency in the absorbances, peak area is to be preferred since this calculation offers a higher reliability than the single wavenumber peak height method. The major advantage of this method is that it is 


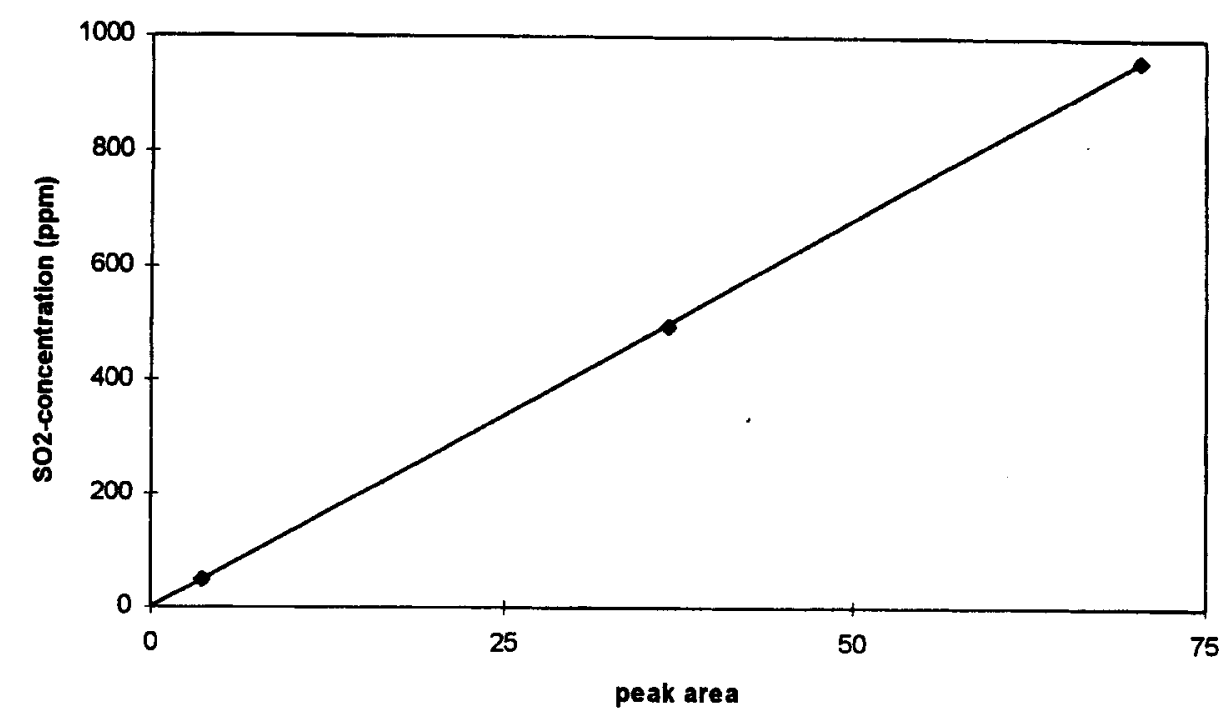

Figure 1. Calibration curve for $\mathrm{SO}_{2}$ : concentration versus peak area in the $1412-1294 \mathrm{~cm}^{-1}$ wavenumber region for a bandwidth resolution of $4 \mathrm{~cm}^{-1}$.

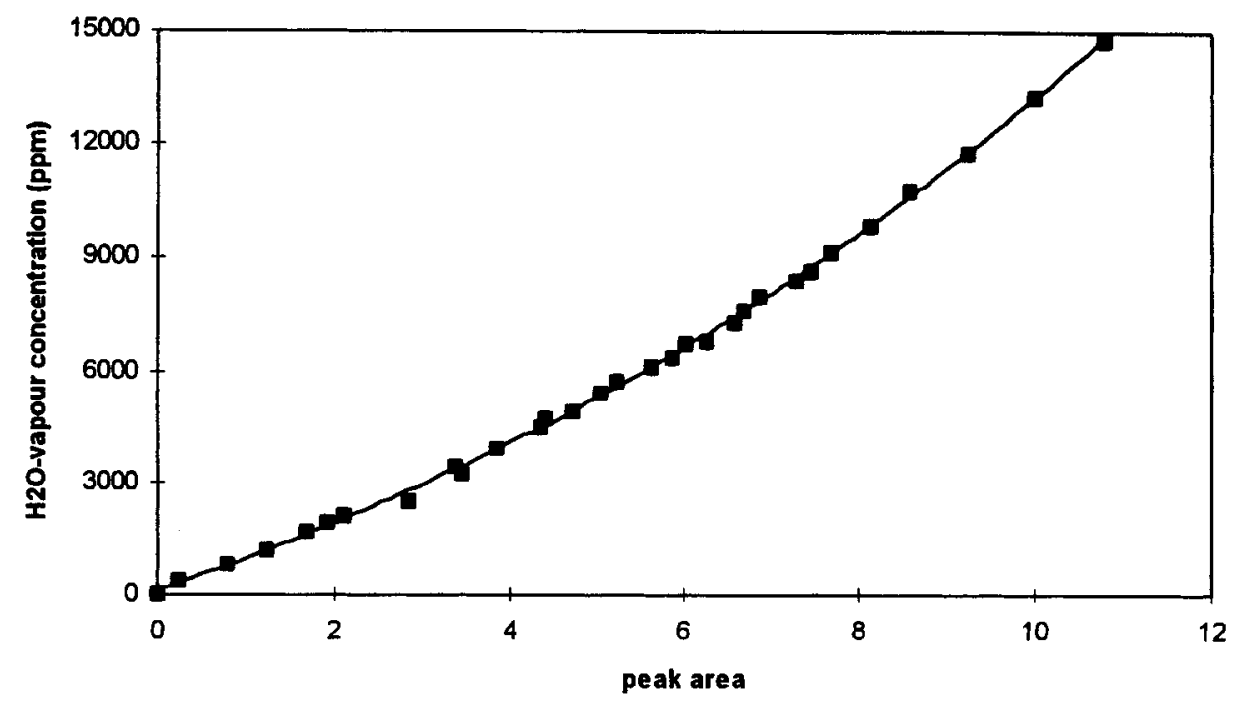

Figure 2. Calibration curve for $\mathrm{H}_{2} \mathrm{O}$-vapour concentration versus peak area in the region $2000-1800 \mathrm{~cm}^{-1}$.

simple and straightforward. In Fig. 1 a calibration curve for $\mathrm{SO}_{2}$, based on the relation between the area calculated in the wavenumber region $1412-1294 \mathrm{~cm}^{-1}$ and the concentration is shown. This area was obtained after baseline correction of the original pure component spectra. The known concentrations were obtained from the supplier of the $\mathrm{SO}_{2} / \mathrm{N}_{2}$ gas mixtures. These concentrations were typically determined after mixing, by the use of GC/MS techniques (gas chromatography/mass spectrometry). This also means that the method which is set up here will always be based on these results and thus on the errors made during the analysis of the supplier. Therefore, even if the relation between peak area and concentration is linear, as is the case for $\mathrm{SO}_{2}$ (and also for other molecules as $\mathrm{C}_{3} \mathrm{H}_{4} \mathrm{O}$ (acrolein)), using more than one gas mixture in the calibration curve can avoid problems coming from erroneous analysis of the supplier. Figure 2 shows that, if the spectra were not collected at full resolution and deviations of Beer's law occur, a nonlinear relation between peak area and concentration can be the consequence. This is not only the case for $\mathrm{H}_{2} \mathrm{O}$ vapour at a resolution of $4 \mathrm{~cm}^{-1}$, but also for molecules such as $\mathrm{HBr}, \mathrm{HCl}, \mathrm{HCN}, \mathrm{CO}, \mathrm{CO}_{2}, \mathrm{NO}, \mathrm{NO}_{2}, \ldots$ which show this typical behaviour at this low resolution. This shows another advantage of the simple peak area method: the method is perfectly capable of dealing with non-linearities, which is not always the case with more complex matrix or full-spectrum methods.

Once the relation between concentration and peak area is put into a mathematical form (a straight line or polynomial fit), predictions can easily be made by calculating the area under the spectral region of interest and applying the mathematical model to predict the unknown concentration.

The major disadvantage of this approach, apart from the fact that it is not really a full-spectrum method, 
is that the method cannot be applied when interfering components are present in the same spectral region. In the case of $\mathrm{SO}_{2}$, the simple straight line is not applicable in most cases in real smoke gas spectra since the region of interest is completely overlapped by the spectrum of water vapour.

\section{Peak area in combination with spectral subtraction}

The peak area method can be applied to spectra with overlapping spectral features if one first succeeds in getting rid of the interfering component. This can be achieved by subtracting (in the case of two interfering components) the pure component spectrum of one component. However, the pure component spectrum first has to be multiplied with a constant factor to obtain the spectrum with the corresponding concentration before subtraction can be applied. This multiplication constant has to be determined by one or another method. In many software packages accompanying FTIR spectrometers, this is still to be achieved by visual inspection, leading to noisy subtraction results. This tool is typically installed to perform qualitative analysis after subtraction. The subtraction result can theoretically be used for quantitative analysis with the peak area method, but large errors are very commonly encountered. Determination of the multiplication constant with a least squares method would be an improvement, but, still, to realize a subtraction result with minor errors, one needs to find a spectral region where only the subtracting component is absorbing, in order to calculate an optimal multiplication constant.

\section{Classical least squares (CLS or K-matrix) ${ }^{7,8}$}

CLS is a multivariate least-squares procedure, directly based on Beer's law. Infrared spectroscopists have sometimes referred to this method as the $K$-matrix method. The CLS model accounts for errors in the spectral measurements. CLS can accommodate spectral intensities at all wavenumbers for all calibration samples. In general, all overlapping spectral components should be known for optimal performance of CLS. By being a fullspectrum method, CLS has the ability to achieve improved precision since there is a signal averaging effect when many or all the spectral intensities are included in the analysis.

Calibration step. The analysis begins with an initial assumption that, at each frequency, there is a linear relation between the concentration $(c)$ of an absorbing species and its spectral absorbance $(A)$. For multicomponent samples, this well-known Beer-Lambert relation (Eqn (1)) takes the form:

$$
A_{i}=\sum_{j=1}^{p} a_{i j} b c_{j}
$$

where $A_{i}$ is the absorbance of a multicomponent sample or reference at a frequency $i, a_{i j}$ is the absorptivity of component $j$ at frequency $i, b$ is the pathlength and $c_{j}$ is the concentration of the $j$ th component ( $p$ components possible in each mixture).
Defining $k_{i j}$ as the product $a_{i j} b$ and adding random error $\left(e_{i}\right)$ in the spectrum at each frequency $i$ yields the following:

$$
A_{i}=\sum_{j=1}^{p} k_{i j} c_{j}+e_{i}
$$

The random error is assumed to be normally distributed with an expectation of zero and a variance proportional to $T^{-2}$ where $T$ is the transmittance value of the spectrum at each wavenumber (absorbance $=\log (1 / T))$.

If a set of accurately known mixtures is used as a reference set, then in the calibration phase of the analysis a series of simultaneous equations can be used to describe the measured mixture spectra. In matrix notation (matrices in bold) these equations are:

$$
\mathbf{A}=\mathbf{C K}+\mathbf{E}
$$

where $\mathbf{A}$ is an $m \times n$ matrix constructed of the absorbance spectra of $m$ mixtures ( $m$ rows) at $n$ frequencies or wavenumbers ( $n$ columns), $\mathbf{C}$ is the $m \times p$ concentration matrix corresponding to the concentrations of each of the $p$ components in the $m$ known references $(m>=p)$. $\mathbf{K}$ is the $p \times n$ matrix consisting of the $k_{i j}$ terms to be estimated by least-squares methods and $\mathbf{E}$ is the $m \times n$ matrix of random errors for each mixture spectrum at each frequency. If Beer's law is valid, then the rows of the K-matrix will be equal to the absorbance spectra at unit concentration and unit pathlength of the $p$ pure component spectra at the $n$ wavenumbers used in the analysis. $\mathbf{K}$ can be obtained by simple matrix manipulations:

$$
\underline{K}=\left(\mathbf{C}^{\prime} \mathbf{C}\right)^{-1} \mathbf{C}^{\prime} \mathbf{A}
$$

$\underline{K}$ is the unweighted least-squares solution to the above set of equations, assuming that $\left(\mathbf{C}^{\prime} \mathbf{C}\right)$ is not singular (this means the inverse could be calculated). The primes indicate transposed matrices. A weighted least-squares estimate of $\mathbf{K}$ can also be obtained by the use of weighting factors which are inversely proportional to the estimated error variance for the infrared spectra in absorbance units. An advantage of the solution given by Eqn (4) is that the entire spectral range of the reference mixture spectra may be used in the estimation of the pure component spectra. Equation (4) shows that the estimate of $\underline{\mathbf{K}}$ is accomplished with a series of matrix multiplication and one matrix inversion. These are readily accomplished with a computer if the dimension of the matrix to be inverted is not large. In this case, the matrix to be inverted, $\left(\mathbf{C}^{\prime} \mathbf{C}\right)$, is a $p \times p$ matrix where $p$ is the number of components.

Since the rows of $\mathbf{K}$ are the estimated spectra of the pure components over the spectral range required for sample analysis, this least-squares estimate will be used to determine the component concentrations in our unknown samples.

Prediction step. If we first assume zero baselines, then the absorbance spectrum of the unknown sample can be expressed by

$$
\mathbf{a}=\mathbf{c K}+\mathbf{e}
$$

where $\mathbf{a}$ is a $1 \times n$ matrix or row vector representing the absorbances of our unknown sample at $n$ wavenumbers; 
c is the row vector containing $p$ component concentrations in the sample; $\mathbf{K}$ is the $p \times n$ matrix representing the pure component spectra and $e$ is the $1 \times n$ row vector representing the error in the sample spectrum (that is, what has not been described by the CLS model).

By using $\mathbf{K}$ from Eqn (4) and from simple matrix manipulations, one obtains the estimate for $\underline{\mathbf{c}}$ by unweighted least-squares:

$$
\underline{\mathbf{c}}=\mathbf{a} \underline{\mathbf{K}}^{\prime}\left(\underline{\mathbf{K K}}^{\prime}\right)^{-1}
$$

assuming the inverse exists.

\section{Baseline corrections}

In what has been described above, we have implicitly assumed zero baselines during calibration and prediction. This is often not the case. However, for the application of smoke gas analysis this is often an almost realistic assumption. Baseline corrections can, however, be made previously to improve accuracy. This can be done in different ways. i.e. one-point baseline correction or twopoint baseline correction. In the one-point baseline correction, the average absorbance is calculated in a region where no absorbing species are present and subtracted from all the absorbance values in the spectral region of interest for that particular component to be evaluated. This assumes a flat and straight baseline which can only be shifted up or down. This seems to be a reasonable assumption for gas analysis. In the two-point baseline correction method, two wavenumbers are chosen at each side of the spectral region of interest and a straight line connecting these two points is used to correct for possible baselineshifts. This corrects for linear deviations in the baseline (translational shifts and tilts).

\section{Non-Beer's law behaviour}

There are a number of instrumental and chemical causes for deviations from Beer's law. The most important instrumental cause stems from the need for monochromatic radiation; i.e. the finite resolution of a spectrometer can lead to a deviation. This will not be too serious for high-resolution instruments, but it can be rather serious for low-resolution ones. Another instrumental deviation is the presence of stray light at the detector.

If there are deviations in Beer's law, then additional changes can be used to help account for these deviations. It is clear that if there are non-linearities in Beer's law, then these non-linearities are approximated in the above multivariate analysis if mixture spectra are used as the initial reference set in the analysis of an unknown sample. This is because the estimated spectra contained in the $\mathbf{K}$ matrix will be the best linear least-squares estimate of the pure component spectra with molecular interactions and spectrometer-related non-linearities included. Of course, the non-linearities are most accurately accounted for if the concentrations of components in the reference mixtures closely surround those of the sample, i.e. the linear nature of Beer's law holds locally. Therefore curvature in the absorbance versus concentration relationship can be approximated ${ }^{9}$ over a limited concentration region by addition of a nonzero intercept term $k_{i o}$, at each wavenumber $i$ to the Beer's law equation, i.e.

$$
A_{i}=\sum_{j=1}^{p} k_{i j} c_{j}+k_{i o}+e_{i}
$$

The $k_{i o}$ term is not only used to approximate non-linear regions of the absorbance versus concentration curves, but will also represent nonzero baselines in the reference and sample spectra. Thus, if the $k_{i o}$ terms are included in the analysis then not only can non-linearities be more nearly approximated, but it is no longer necessary to make preliminary corrections for these constant nonzero baselines.

In practice, the explicit least-squares estimate of the deviation of linearity can be obtained by the addition of an extra row of $k_{i o}$ terms $(i=1$ to $n)$ to the bottom of the $\mathbf{K}$ matrix and a column of 1's in the concentration matrix $\bar{C}$ (in fact, these must be the inverses of the reference pathlengths if there is reason to assume that pathlength variations are possible). The K-matrix then becomes a $(p+1) \times n$ matrix and $\mathbf{C}$ a $m \times(p+1)$ matrix. In what follows, the first CLS method (without $k_{i o}$ terms) is referred to as CLSI, the second method, including the $k_{i o}$ terms is called CLSII.

\section{Statistical precision of the estimated concentration}

It is important to be able to assess the statistical precision in the estimated concentrations. Note, however, that the statistical error in $\mathbf{C}$ is a non-linear function of the errors in the estimated matrix $\mathbf{K}$ and the errors in the sample spectrum a. Thus, calculation of standard errors of the estimated concentrations is not at all straightforward. One approach which can be taken, if time and resources permit, is to repeat the measurement of the reference mixture spectra and the sample spectrum, along with the calculation of $\underline{\mathbf{C}}, r$ times. The variations of these estimates across the $r$ replications could be used to obtain standard errors and statistical confidence limits.

\section{Selection of standard mixtures and number of wavenumbers}

Certain precautions should be followed in selecting standard mixtures for determining the K-matrix. The concentration range should encompass all possible unknown concentrations; e.g. the highest concentration of an unknown should be less than the highest concentration in a standard mixture. Furthermore, the magnitude of the concentrations of the standards and unknown should be similar; e.g. standards in the range of 0 to $1000 \mathrm{ppm}$ should not be used for unknowns in the ppb region even though the ppb range is included in the concentration range of the standards. Both these precautions are necessary because of possible deviations from Beer's law.

Another precaution has to be considered. The relative concentration of components in each standard mixture must be different. If two standard mixtures have the same relative concentrations, one row of the $\mathbf{C}$-matrix will be proportional to another row. The matrix is then singular and the matrix inverse of $\mathbf{C}^{\prime} \mathbf{C}$ will not exist. 
The number of standards and/or the number of wavenumbers no longer causes computer calculation problems as was the case ten years ago. However, a reasonable choice of these numbers is still advisable in order not to overdetermine the absorbance matrix. If, for instance, $\mathrm{SO}_{2}$ absorbs in the wavenumber region $1412-1294 \mathrm{~cm}^{-1}$, then it makes no sense to add absorbances to the calibration matrix from wavenumber regions outside this region of interest. The choice of the standards has to be such that they are uniformly distributed in the 'concentration space'.

\section{The use of CLS-residuals}

What is not described by the CLS model is contained in the residual spectrum e. Full-spectrum residuals can be useful for determining the presence and possibly the identity of unexpected components. Features in the residual spectra that are greater than the noise indicate the presence of model errors. Model errors can arise from non-linearities or unknown components that are not adequately accounted for during the CLS calibration. If impurities are not present in the calibration samples, but are present in the unknown ones, then the impurity bands would be primarily positive in the residual spectra of the unknown samples. The relative magnitude of the residuals in the unknown samples can be used for detection of outlier samples. The ability to examine full-spectrum residuals in CLS can yield chemically interpretable information concerning the presence and identity of unknown or unexpected components.

\section{Advantages and disadvantages of CLS}

Since CLS is a full-spectrum method, it can

(1) Provide significant improvements in precision over methods that are restricted to a small number of frequencies.

(2) Allow simultaneous fitting of spectral baselines and correct for small non-linearities

(3) Make available for examination and interpretation least-squares estimated pure-component spectra and full-spectrum residuals.

A major disadvantage of the CLS method is that all interfering chemical components in the spectral region of interest need to be known and included in the calibration.

\section{Kalman filter}

The Kalman filter is a recursive method which can be used for calibration and prediction of multicomponent spectra. It is a digital filtering algorithm which allows the estimation of states and parameters from noisy measurements. ${ }^{10}$ Kalman filter methods may be advantageous for multicomponent analysis in the presence of unanticipated and unmodelled responses in a multicomponent signal. The Kalman filter is often used as a prediction algorithm in combination with CLS calibration. ${ }^{11}$ However, it can be used for calibration and prediction as well. ${ }^{12}$ The Kalman filter method is, in fact, a way to alter
CLS to perform the regression of the unknown response onto models sequentially, rather than in a single step. While sequential regression may not always be as computationally efficient as ordinary regression, it can sometimes be more computationally efficient, depending on the number of parameters to be fitted, the dimension of the measurement and the weighting factors. Sequential regression also offers other advantages. Two of these advantages are the elimination of the need for matrix inversion and the possibility of using prior information on the values and/or distribution of the concentration matrix and the covariance matrix, which is calculated in this method. However, in a comparative study ${ }^{13}$ calibration-based inverse regression methods (like PCR) offered improved methods of filter design when the signal and noise characteristics of a system are not totally known and when complex properties are being estimated.

\section{Inverse Least Squares (ILS or P-matrix) ${ }^{14}$}

The inverse least squares method is also known as the P-matrix method or as Multiple Linear Regression (MLR). The inverse least squares method is in fact based on the inverse of Beer's law. For $m$ calibration standards, one can write the inverse of Beer's law in matrix form:

$$
\mathbf{C}=\mathbf{A P}+\mathbf{E}
$$

where $\mathbf{C}$ and $\mathbf{A}$ are defined as before and $\mathbf{P}$ is the $n \times p$ matrix of the unknown calibration coefficients relating the $p$ component concentrations to the spectral intensities. $\mathbf{E}$ is the $m \times p$ matrix of random concentration errors or residuals that are not fit by the model. This model thus minimizes the squared errors in concentration during calibration. The inverse representation of Beer's law has the significant advantage that the analysis is invariant with respect to the number of components $p$ in the analysis. This means that we can write:

$$
\mathbf{c}=\mathbf{A p}+\mathbf{e}
$$

Here $\mathrm{c}$ is the $m \times 1$ column vector of concentrations of the component of interest in the $m$ calibration spectra, $\mathbf{p}$ is the $n \times 1$ vector of the calibration coefficients and $e$ is again the $m \times 1$ vector of concentrations residuals not fit by the model.

During calibration, the least-squares solution for $\mathbf{p}$ in Eqn (9) is:

$$
\underline{\mathbf{p}}=\left(\mathbf{A}^{\prime} \mathbf{A}\right)^{-1} \mathbf{A}^{\prime} \mathbf{c}
$$

During prediction, the solution for the component concentration in the unknown sample spectrum is simply

$$
c=\mathbf{a}^{\prime} \underline{\mathbf{p}}
$$

This means a quantitative spectral analysis can be performed even if the concentration of only one component is known in the calibration mixtures. The components for which the concentrations are not included in the analysis must be present in the calibration spectra and are implicitely modelled during calibration. This advantage of ILS has resulted in it being used for NIR (near-infrared) analysis methods, where it is known as MLR (multiple linear regression). 
A disadvantage of ILS is that the analysis is generally be restricted to a small number of wavenumbers. This is because the matrix which must be inverted in Eqn (10) has the dimension equal to the number of wavenumbers and this number cannot exceed the number of calibration mixtures used in the analysis. So there must always be at least as many samples or spectra as there are wavenumbers present in the absorbance matrix. In addition, this can give rise to what is known as collinearity problems (or zero determinant or singularity, all names for the same problem; i.e. the near-linear relationships between absorbances at multiple wavenumbers). In this case the matrix inversion is impossible or becomes cumbersome. These problems can be especially significant if the number of wavenumbers becomes too large and the precision of the results is actually degraded when too many wavenumbers are included in the analysis. As a consequence, another problem arises when one has to determine how many and which wavenumbers must be included in the analysis. As stated above, the ILS or MLR model has been very popular among NIR fixed filter equipment users, but one sees that, increasingly, with the new multiple wavenumber monochromator systems, PLS (Partial Least Squares), which is based on PCA (Principal Component Analysis), becomes an alternative for MLR.

\section{Q-matrix ${ }^{15}$}

A third form of simple multiple linear regression covering full-spectrum analysis is called the $Q$-matrix method, analogously to $K$ - and $P$-matrix methods. This method has also been proposed by Nyden and Babrauskas ${ }^{1}$ for smoke gas analysis. The fundamental hypothesis of the $Q$-matrix approach is that the spectrum of an unknown mixture can be synthesized from a linear combination of the spectra of the components included in the mixture. The mathematical expression of this is

$$
\mathbf{u}=\mathbf{q} \mathbf{A}
$$

where $\mathbf{u}$ is the row vector $(1 \times n)$ containing the absorbance spectrum of the unknown, $q$ is the row vector $(1 \times m)$ containing the regression coefficients which provide the least squares fit of the unknown spectrum by the standard spectra and $\mathbf{A}$ is the $(m \times n)$ absorbance matrix of $m$ calibration spectra at $n$ wavenumbers. This is conceptually the reverse of the process of multiple spectral subtraction in which the spectra of the respective components are subtracted from the spectrum of the mixture with the appropriate factors until the final residue spectrum is composed of just noise. The form of the generalized solution of Eqn (12), during the calibration step, is:

$$
\mathbf{q}=\mathbf{u} \mathbf{A}^{\prime}\left(\mathbf{A A}^{\prime}\right)^{-1}
$$

where $\mathbf{A}^{\prime}$ is the transposed matrix of $\mathbf{A} . \mathbf{A} \mathbf{A}^{\prime}$ is an $m \times m$ matrix which must be inverted. An immediate consequence of this is the dimensional constraint that the number of wavenumbers has to be higher than the number of calibration samples or the system is underdetermined and the inverse cannot be calculated. This is, of course, a major drawback of the method if the spectral region of interest is rather restricted, which is often the case for individual gas components in FTIR spectra.
Within the limits of this description, the idea of concentrations does not enter into the initial least squares solution. When mixtures are used as calibration standards, the Beer's law non-linearities are effectively incorporated into the solution of the $Q$-matrix, which is, of course, one of the advantages of this method. The values of the component concentrations in the unknown sample are easily derived during the prediction step from the $Q$-matrix by multiplying the $\mathrm{C}$-matrix as in

$$
\mathrm{d}=\mathbf{q} \mathbf{C}
$$

where $\mathbf{d}$ is the row vector $(1 \times p)$ containing the concentrations of the $p$ components in the unknown spectrum, $q$ is the $(1 \times m)$ solution from Eqn (13) and $\mathbf{C}$ is the $(m \times p)$ concentration matrix of the calibration samples. It is clear that Eqns (13) and (14) can be combined for a direct solution of the concentration values to be determined. Theoretically, however, Eqn (13) implies that the matrix inversion in Eqn (13) need be calculated only once, during the calibration step.

Due to noise, a variant of this method has been proposed, incorporating a weighting function into Eqn (12). The major drawback of this method is that each time the concentrations of the unknown have to be predicted, an analogous matrix inversion as in Eqn (13) has to be realized. Even with a very fast personal computer, and depending on the rank of the matrix, this inversion can take some time and consequently the method is not very suitable for on-line purposes or for the analysis of many smoke gas fire spectra as in the case of, for example, a cone calorimeter/FTIR test.

Principal Component Analysis ${ }^{16,17} /$ Factor Analysis $^{18-20}$

Although not completely the same, factor analysis (FA) and Principal Component Analysis (PCA) form the basis of Principal Component Regression (PCR) and Partial Least Squares (PLS). In PCA or FA a decomposition of the original absorbance matrix $\mathbf{A}$ is realized into two new matrices, called scores matrix $\mathbf{T}$ and loadings matrix $\mathbf{P}$ (we will focus on PCA terminology in what follows):

$$
\mathbf{A}=\mathbf{T P}^{\prime}
$$

where the prime in Eqn (15) denotes the transposed of the matrix. Each row in $\mathbf{T}$ and $\mathbf{P}$ consists of elements, called scores and loadings respectively, constituting score vectors $\mathbf{t}$ and loading vectors $\mathbf{p}$.

PCA performs, in fact, a coordinate transformation into a new coordinate system. In the old coordinate system, each column in the absorbance matrix $\mathbf{A}$ referring to a wavelength, corresponds to a coordinate axis. A spectrum in this multidimensional space can be represented by a simple point. In the new coordinate system, the loading vectors $\mathbf{p}$ are the new basis vectors, that is, orthonormal vectors (having unit length and being orthogonal to each other). The direction of these loading vectors is along the new axes, which are called principal components. The elements $p_{i}$ of the loading vectors are the direction cosines or the projections of the unit vector along the principal component on the $i$ th axis of the old coordinate system. The element $t_{i}$ of the scores vectors are the coordinates of the $i$ th point (ith spectrum) on the new coordinate axes (= principal components). The 
transformation is calculated such that the new coordinate axes are lying in directions of maximum variance. The aim of PCA is to restrict the number of coordinate axes, thus reducing the dimensionality of the problem. If one has originally, say, 50 wavelengths then the old coordinate axes construct a 50-dimensional space in which $100 \%$ of the variance in the spectral data is represented. After PCA, it is very common to find that a limited number of two or three principal components suffice to explain over $99 \%$ of the variation in the spectral data. This means that a reduction of the dimensionality from 50 to 2 or 3 is achieved in this way. The $1 \%$ left is often not relevant and can be considered as spectral noise or residuals. Therefore, the result of the decomposition of the original absorbance matrix A after PCA is given by

$$
\mathbf{A}=\mathbf{T} \mathbf{P}^{\prime}+\mathbf{E}
$$

where $\mathbf{E}$ is a matrix containing the spectral residuals.

Calculation of the different principal components is obtained by using the NIPALS algorithm (Non-linear Iterative PArtial Least Squares). It does not calculate all the principal components at once. It calculates the vectors $\mathbf{t}_{1}$ and $\mathbf{p}_{\mathbf{1}}{ }^{\prime}$ from the A-matrix. Then the production $\mathbf{t}_{\mathbf{1}} \mathbf{p}_{1}^{\prime}$ is subtracted from the A-matrix and the residual $\mathbf{E}_{1}$ is calculated. This residual is then used to calculate $\mathbf{t}_{\mathbf{2}}$ and $\mathbf{p}_{2}{ }^{\prime}$, etc. NIPALS is an efficient iterative algorithm which extracts the full spectrum loading vectors from the spectra in the order of their contribution to the variance in the calibration spectra. This means that the first principal component will explain as much of the variance as possible, the second principal component will explain as much of the variance as possible of what is not explained by the first one, etc.

From what is explained above, it must be clear that PCA works on the absorbance matrix $\mathbf{A}$ and has nothing to do with the concentration matrix $\mathbf{C}$. A regression technique relating the scores and the concentrations is called Principal Component Regression (PCR). The potential problem with PCR is that the loading vectors which best represent the spectral data may not be optimal for concentration prediction. Therefore, it would be desirable to derive loading vectors so that more predictive information is placed in the first few principal components.

\section{Partial Least Squares ${ }^{6,14,17,21}$}

The PLS model is built on the properties of the NIPALS algorithm. In fact, the PLS algorithm is a modification of the NIPALS algorithm and it achieves the goal to place more predictive information in the first few PCs by using concentration information to obtain the decomposition of the spectral matrix A. Concentration-dependent loading vectors are generated and the computed scores $\mathbf{T}$ are then related to the concentrations or concentration residuals after each loading vector is calculated.

In the calibration step, the absorbance matrix $\mathbf{A}$ and the concentration matrix $\mathbf{C}$ are decomposed in

$$
\begin{aligned}
& \mathbf{A}=\mathbf{T} \mathbf{P}^{\prime}+\mathbf{E} \\
& \mathbf{C}=\mathbf{U} \mathbf{Q}^{\prime}+\mathbf{F}
\end{aligned}
$$

Regression of the scores $t$ against the scores $u$ results in a relation between them:

$$
\mathbf{U}=\mathbf{T B}
$$

where B contains the regression coefficients for regression of $t$ against $u$. Equation (19) is often referred to as the inner relation between $A$ and $C$ scores. Equation (19) assumes a linear relation between scores $t$ and $u$. If this is not a good approximation, then there exists a non-linear relationship between them and this can be observed by plotting the scores $t$ versus $u^{22}$ Non-linear PLS is then the appropriate model to choose. The mixed relation is:

$$
\mathbf{C}=\mathbf{T B Q}^{\prime}+\mathbf{F}
$$

where the concentration residuals $F$ have to be minimized in a least squares sense. This presentation of PLS does not give the best possible model. The reason is that the principal components are calculated for both $\mathbf{A}$ and C separately so that they have a weak relation to each other. It would be better to give them information about each other so that slightly rotated components result which lie closer to the regression line. To improve this relation $t$ and $\mathbf{u}$ scores are exchanged.

It is not the purpose to give all the details of how the PLS algorithm exactly calculates scores, loadings and regression coefficients. Excellent papers dealing with this topic are from Geladi ${ }^{21}$ and Haaland. ${ }^{14}$ Also Martens and Naes ${ }^{17}$ present PCA and PLS algorithms.

The intention of PLS is to describe $\mathbf{C}$ as well as is possible and hence to make $\mathbf{F}$ as small as possible (in a least squares sense), and at the same time get a useful relation between $\mathbf{A}$ and $\mathbf{C}$. An important point to note is that the algorithm does not give orthogonal $t$-values (as is the case in PCA and PCR). The reason is that the order of calculations that was used for the PCA has been changed. Therefore, the loading vectors $\mathbf{p}^{\prime}$ are replaced by loading weights $\mathbf{w}^{\prime}$, and an extra loop has to be included to get orthogonal $t$-values. Orthogonal $t$-values are not absolutely necessary but they make the comparison with PCR easier.

With PLS it is possible to calibrate or analyse two or more components at the same time, by using a global PLS algorithm called PLS2. ${ }^{21}$ However, PLS1 has been found to have better predictive abilities than PLS2. ${ }^{14}$

The important part of any regression is its use in the prediction step. We here assume that the optimal number of PLS components has been chosen to obtain the best model for prediction. PLS prediction can be obtained by applying two different methods.

The first method for obtaining concentration predictions involves the calculation and use of the vector of final calibration coefficients $b$. The b-vector need only be calculated once from the calibration results. Although the b-vector provides an efficient method to estimate concentrations from any unknown sample spectrum, it does not allow a determination of the residual spectrum. Therefore, no diagnostic information about the quality of the fit is available when predictions are obtained by using the $\mathbf{b}$-vector.

The second method is more involved but allows spectral residuals to be calculated. This method requires the calculation of the scores of the sample spectrum; that is, the representation of the spectrum in the new coordinate 
system constructed by the PLS PCs. From the calibration loadings and loadings weights matrix and with the new scores, it is then possible to calculate a prediction value for the unknown sample. Moreover, full spectrum residuals are calculated at the same time. These residuals can be useful for determining the presence and possibly the identity of unexpected components. However, identifying unexpected components occurs more rapidly for a spectrum obtained in the mid-infrared region, where spectral bands are more characteristic on a molecular level.

\section{PLS-residuals used as a diagnostic system ${ }^{21,17,14}$}

Discrepancies of many different kinds between a model and the data can be detected by studying residuals. These residuals are the quantities remaining after the systematic contributions associated with the assumed model are removed. When assumptions concerning the adequacy of the model are true, we expect to find that the residuals vary randomly. If, however, we find that the residuals contain unexplained systematic tendencies, we shall be suspicious of the model or, during prediction, of the unknown sample to be analysed. It is therefore always interesting to calculate the residuals during prediction and compare them to those obtained from the calibration samples, that is, the samples that were used for model building. The most common form of discrepancy revealed by comparison of calibration model residuals with residuals coming from an unknown sample occurs when the residuals of the latter are much larger than the calibration residuals. But there are also other diagnostic possibilities when the elements of the residual matrix $\mathbf{E}$ are subjected to a close inspection. Sums of squares over the columns of $\mathbf{E}$ indicate the importance of a variable (wavelength) for a certain component. Sums of squares over the rows of $\mathbf{E}$ indicate how well the samples (objects) fit the model. This can be used as an outlier detection criterion.

An advantage of PLS, like CLS, is that these statistics can be calculated for every component. These statistics can thus be used to estimate which objects and variables contribute mainly to the model and which contribute mainly to the residual.

$F$-statistics can be used as an outlier detection criterion. Indeed, during calibration, outliers can be detected both from concentration $F$-ratios or spectral $F$-ratios while outlier detection in the unknown samples must rely solely on the spectral $F$-ratios. The spectral $F$-ratio can be defined as:

$$
F=m \sum_{j} e_{i j}^{2} / \sum_{i} \sum_{j} e_{i j}^{2}
$$

where the summation over $j$ is over the wavenumbers and the summation over $i$ is over the calibration samples and $m$ is the number of calibration samples. The spectral $F$-ratio is thus the sum of squared spectral residuals for the fit of the unknown sample spectrum to the average of the sum of squared residuals of the calibration spectral estimates. We expect the $F$-ratio to be close to 1 . The question now is: how much larger should this be before the object is considered suspicious? There is no exact answer to this, but one can conclude that the object is suspicious if the quantity is larger than a certain percentile in the $F$-distribution. However, for IR spectral data where the error is dependent on the absorbance levels, spectral evidence suggested that spectral $F$-ratios less than 3 or perhaps be 5 do not appear to indicate a highly significant outlier. As a rough and practical approach one can state that $F$-ratios above 5 might be appropriate to flag outlier samples with respect to the original calibration data set.

\section{Quantitative validation and rank selection for PLS calibration ${ }^{23}$}

The optimal number of PCs (or the optimal pseudo-rank) which gives the optimal calibration model is chosen, based on a validation statistic. Since the calibration models are used to predict the concentrations of the analyte in future samples, a prediction error statistic is calculated. If additional samples and concentration information for these samples are available (independent test set), the rootmean-square error of prediction (RMSEP) can be found by

$$
\text { RMSEP }=\sqrt{\frac{\sum_{i=1}^{m}\left(c_{i}-c_{i}^{*}\right)^{2}}{m}}
$$

where $m$ is the number of samples in the test set. These samples were not used in the model building step. $c_{i}$ are the independently determined concentrations and $c_{i}^{*}$ are the predicted concentrations. As $m$ becomes large the RMSEP will approach the prediction error of the population of all future samples. Other statistical parameters can be used to define the 'goodness' of the model, i.e. the Standard Error of Prediction and the bias (estimate for a systematic error):

$$
\mathrm{SEP}=\sqrt{\frac{\sum_{i=1}^{m}\left(c_{i}-c_{i}^{*}-\mathrm{bias}\right)^{2}}{m-1}}
$$

where

$$
\text { bias }=\frac{\sum_{i=1}^{m}\left(c_{i}-c_{i}^{*}\right)}{m}
$$

which is defined as the average difference between measured and predicted concentrations. Later one can evaluate the slope, offset and correlation coefficient for the regression line in the plot of the predicted versus reference concentration of the test set samples.

If only the calibration data are available, another approximation to the prediction error is often calculated, using a method called cross-validation. In this method, some of the data are omitted, a model is built with the remaining data and then a prediction is made using the omitted data. This is repeated until all the data have been omitted once. The most commonly applied method in calibration is called leave-one-out cross-validation, where one sample is left out at a time. The corresponding statistics, the root-mean-square error of cross-validation (RMSECV), is

$$
\text { RMSECV }=\sqrt{\frac{\sum_{i=1}^{n}\left(c_{i}-c_{i}^{*}\right)^{2}}{n}}
$$


where $n$ is the number of calibration samples and $c_{i}^{*}$ is the predicted concentration when a model is built without sample $i$. Cross-validation is often used instead of prediction since it is usually difficult to obtain enough prediction samples to make RMSEP statistically significant. However, cross-validation is computationally expensive since $n$ calibration models must be calculated.

The selection of pseudo-rank is important because the model should describe the significant sources of variance but not overfit the data. A model is overfitting if it includes non-relevant information in the model from the calibration set, such as non-random components in the noise or fluctuations due to experimental conditions. A model that is overfitting will have poorer prediction errors. Several schemes have been proposed for model selection given the RMSECV at different choices of the pseudo-rank. The simplest criterion is to choose the pseudo-rank corresponding to the first minimum in a curve of pseudo-rank versus RMSECV. Further theories have been developed beyond this, because since the RMSECV is calculated with a finite number of samples, there is an error associated with it.

All these theories use the variance term from RMSECV (which is a standard deviation), called PRESS (Predictive Residual Error Sum of Squares):

$$
\text { PRESS }=\sum_{i=1}^{n}\left(c_{i}-c_{i}^{*}\right)^{2}
$$

One of these theories is given by Haaland and Thomas. ${ }^{14}$ They propose to calculate an $F$-statistic with $(n, n)$ degrees of freedom:

$$
F(r)=\frac{\operatorname{PRESS}(r)}{\operatorname{PRESS}\left(r^{*}\right)}
$$

where $r^{*}$ is the pseudo-rank, that yield the minimum PRESS. This statistic tells if the two PRESS values are significantly different. The optimal pseudo-rank is the smallest value of $r$ that has a PRESS which is not significantly different from PRESS $\left(r^{*}\right)$.

\section{INLR, implicit non-linear latent variable regression ${ }^{22}$}

One of the major problems with all the full-spectrum methods cited above is that they are not able to handle large non-linear deviations from Beer's law. Small nonlinearities can be covered by CLSII, PCR, PLS and Q-matrix. Although several attempts have been made to construct algorithms for non-linear $\mathrm{PLS}^{24,25}$ the approaches were always very complicated, demanding elaborate programming. A very simple approach, called INLR, ${ }^{22}$ to handle non-linearities is by adding squared variables to the absorbance matrix. In that case, both the square and cross terms of the latent variables are implicitly included in the resulting PLS model. This means that it is not necessary, in this approach, to include the cross-terms of the absorbance variables. Analogously, with cubic non-linearities expanding the A-matrix with cubic terms $a_{i j}^{3}$ is sufficient. INLR is attractive in that all essential features of PLS are preserved, i.e. (1) it can handle many noisy and collinear variables, (2) it is stable and gives reliable results and (3) all PLS plots and diagnostics still apply.

\section{Artificial neural networks (ANNs) ${ }^{26}$}

For completeness, a few words have to be said about ANNs. The field of neural networks covers a wide range of different network methods which are developed for and applied to very different situations. In particular, the 'feedforward' network structure is suitable for handling non-linear relationships between input and output ${ }^{27}$ variables when the focus is prediction. Reference 27 also discusses the differences between feedforward and other networks. Neural nets have already been applied successfully in calibration problems with spectroscopic data. ${ }^{28,29}$ One of the most popular ANN algorithms is the backpropagation of errors learning method. Again, here a learning, training or calibration step is required before prediction of unknown samples can be realized. However, for the learning procedure, ANN is quite different from PCR or PLS. Since the ANN method concentrates on fitting without restrictions, there are reasons to believe that ANNs are more sensitive to overfitting than are PLS and PCR. As a compromise, it has been proposed to use principal components as input for the network instead of the original variables themselves. ${ }^{30}$ The training or learning step in the calibration procedure with ANNs consists of presenting the absorbances or PLS scores to the input layer of the net in a random or sequential order. An ANN consists of so-called neurons, which are organized in layers. The organization is such that a neuron in a specific layer is connected to all neurons in the previous layer and to all neurons in the next layer. A simple ANN consists of one input layer, one hidden layer and one output layer. The input layer is the first layer of the net and does nothing more than distribute each set of absorbances or PLS scores to the next layer. The flow of information through the net is such that each neuron receives signals from neurons in the previous layers and sends signals to neurons in the following layer. The output of the net is calculated and compared with the reference. The difference between the reference and the value predicted by the net is the error. The error is minimized by adjusting weights and the bias of each neuron. The direction in which this is done is from output layer towards input layer (backpropagation). When this error is sufficiently small for all samples in the training set, the network has completed its training phase and is ready for testing or prediction. A major advantage of ANNs is that they can handle non-linearities, but they are often sensitive to overfitting.

\section{EXAMPLES OF MULTIVARIATE SMOKE GAS ANALYSIS}

All spectra on the gas mixtures were collected at a resolution of $4 \mathrm{~cm}^{-1}$ and $150^{\circ} \mathrm{C}$, using continuous flow between 2 and $61 \mathrm{~min}^{-1}$. The gas mixtures used for calibration were continuously drawn from gas cylinders, supplied with a certificate (exact concentration determined by GC/MS by the supplier), via a heated transfer line, through a heated gas cell (pathlength: $3.2 \mathrm{~m}$ ) in the spectrometer (Nicolet Magna 550). Examples will be given for the quantitative analysis of $\mathrm{SO}_{2}$ in the region 


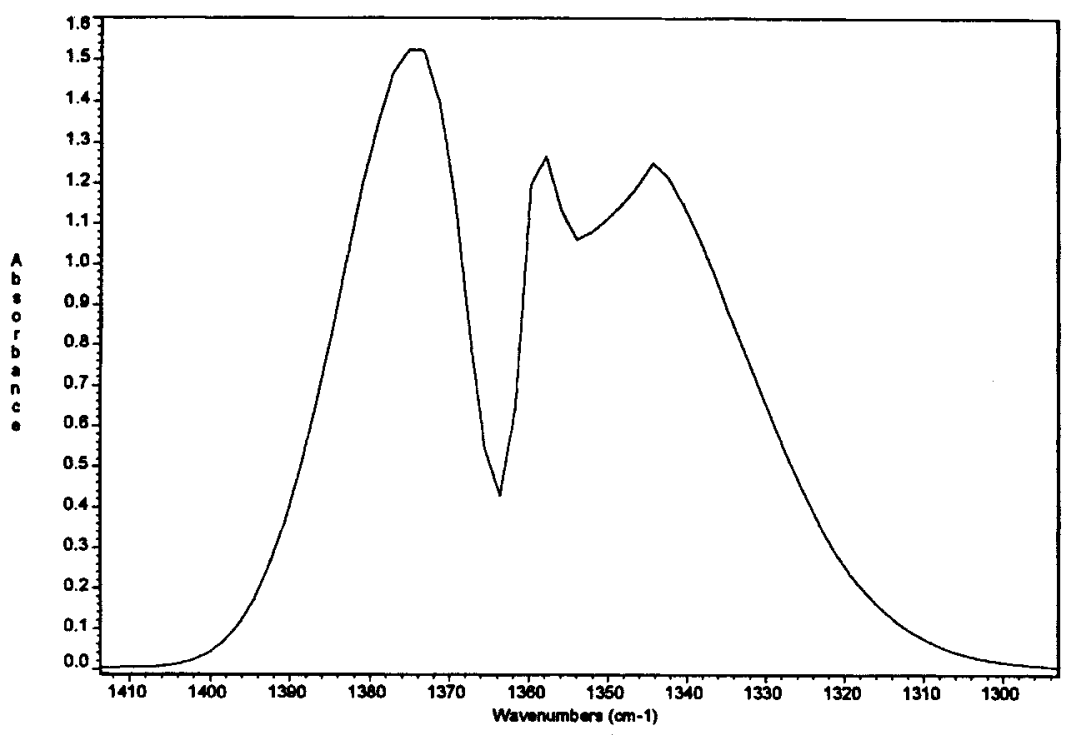

Figure 3. The spectrum of $\mathrm{SO}_{2}(960 \mathrm{ppm})$ in nitrogen.

$1412-1294 \mathrm{~cm}^{-1}$ and $\mathrm{CO}_{2}$ in the region $3763-3500 \mathrm{~cm}^{-1}$. Water vapour absorbs in both regions. Totally overlapping spectral bands cannot be quantitatively resolved by simply using peak height or peak area. Spectral subtraction in combination with peak area or full-spectrum methods can be used. Calibration sets were constructed from three pure $\mathrm{SO}_{2}$ spectra (Fig. 3; concentrations were 50,497 and $960 \mathrm{ppm}$ ) and ten pure $\mathrm{H}_{2} \mathrm{O}$ spectra (concentrations between 368 and $8500 \mathrm{ppm}$ ). From the three pure $\mathrm{SO}_{2}$ spectra, 28 pure $\mathrm{SO}_{2}$ spectra were constructed, by multiplying these three spectra with different constants and using the calibration curve given in Fig. 1 to determine the concentration.

Software used for the calculation of calibration models and prediction is mainly self-written (Microsoft Visual Basic which allows compatibility with the 'Omnic' software of the Nicolet spectrometer). Also 'The Unscrambler' (Camo) has been used for some PLS models.

\section{CLSI-model for $\mathrm{SO}_{2}$}

A CLS model was calculated using 280 mixture spectra (28 pure $\mathrm{SO}_{2}$ spectra were added to 10 pure $\mathrm{H}_{2} \mathrm{O}$ spectra; $\mathrm{SO}_{2}$ range: $5-960 \mathrm{ppm}, \mathrm{H}_{2} \mathrm{O}$ range: $368-8500 \mathrm{ppm}$ ). Each mixture spectrum was baseline corrected with a onepoint baseline correction calculated in the region $2650-2600 \mathrm{~cm}^{-1}$, before it was added to the calibration absorbance matrix $\mathbf{A}$. This matrix contained 280 rows and 64 columns, corresponding to the 280 reference spectra with varying concentrations of $\mathrm{SO}_{2}$ and $\mathrm{H}_{2} \mathrm{O}$ and to 64 wavenumbers between $1412-1294 \mathrm{~cm}^{-1}$.

The concentration matrix $\mathbf{C}$ contained 280 rows and 2 columns, corresponding to the 280 reference spectra and their respective concentrations for $\mathrm{SO}_{2}$ and $\mathrm{H}_{2} \mathrm{O}$. From (4), the K-matrix solution during calibration was calculated and is shown in what is called the Kspectrum (Fig. 4). For comparison, the pure $\mathrm{SO}_{2}$ spectrum with a concentration of $960 \mathrm{ppm}$ is shown as well (Fig. 3).
With the obtained K-matrix solution, the calibration samples were predicted and the predicted versus reference concentrations are shown below, for both components $\mathrm{SO}_{2}$ and $\mathrm{H}_{2} \mathrm{O}$. Calibration statistics, given by the Root Mean Square Error of Calibration (RMSEC, definition as in Eqn (22a) with $m$ the degrees of freedom), for $\mathrm{SO}_{2}$ is $\mathrm{RMSEC}=1.41 \mathrm{ppm}$ and for $\mathrm{H}_{2} \mathrm{O}$ is RMSEC $=422.9 \mathrm{ppm}$. Excellent calibration results were obtained for $\mathrm{SO}_{2}$ (Fig. 5). The non-linear nature of the concentration-absorbance dependence for $\mathrm{H}_{2} \mathrm{O}$ is clearly visible in the predicted versus reference graph (Fig. 6). CLSI is not able to deal with these nonlinearities.

From Eqn (3b) one can see that the E-matrix can be calculated for the calibration samples after introducing the K-solution in the equation. Each row in the E-matrix represents the residual spectrum for the corresponding reference spectrum; that is, what has not been described by the CLS model. The magnitude of the sum of squares of these residuals gives an indication of the goodness of the fit and/or for the presence of possible outlying samples during calibration. The average sum of squares (over the columns) of the residuals for all the calibration samples is useful for later comparison with the sum of squares of the residuals of an unknown sample predicted with the model. The average sum of squares of residuals for all the 280 samples is here $8.9 \times 10^{-4}$. This value can be used for comparison with the sum of squared residuals of future unknown samples ( $F$-statistics, Eqn (21)). If we use this value for the calibration set and plot it for each calibration spectrum, then we obtain Fig. 7.

The average $F$-value is of course equal to 1 . In the calibration set, $F$-values up to 6 were found, without really finding a reason to omit samples in the calibration. From the literature it is known that $F$-values of 3 or less do not indicate outlying samples. However, here we see that $F$-values can be up to 6 without really indicating a sample as outlying.

The sum of squared residuals calculated for each column (that is summed over the rows) gives information on 


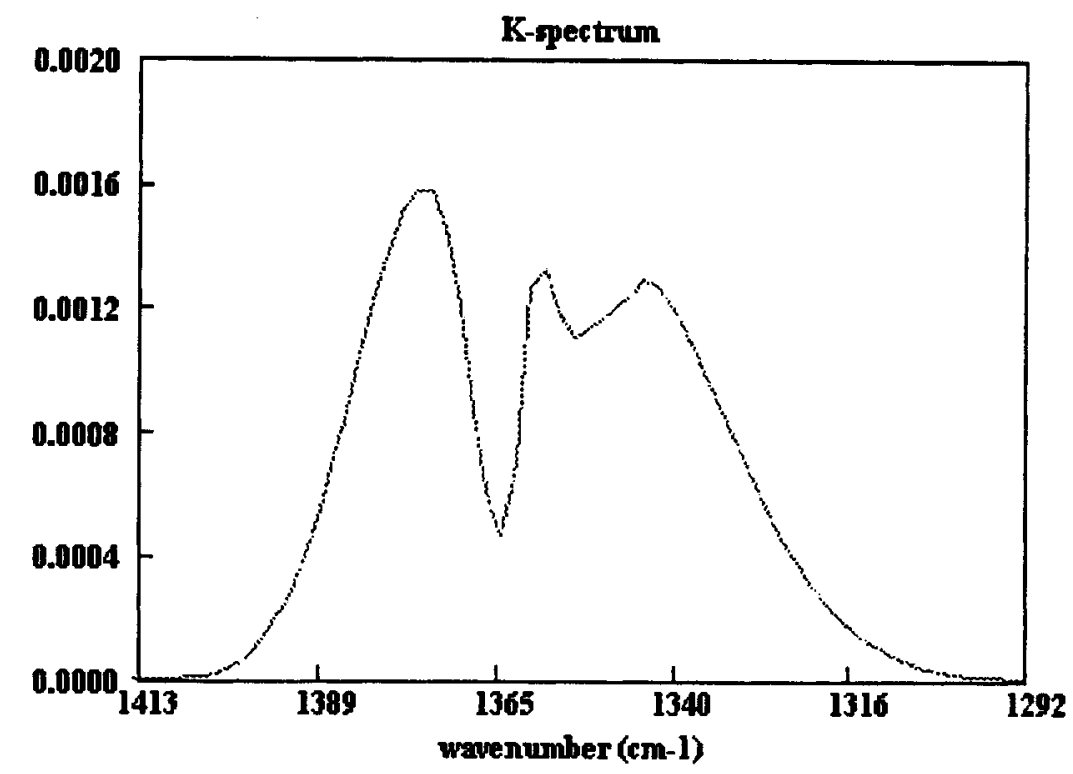

Figure 4. $\mathrm{K}$-spectrum for $\mathrm{SO}_{2}$ obtained after $\mathrm{CLSI}$ calibration (unlabelled ordinate in arbitrary units).

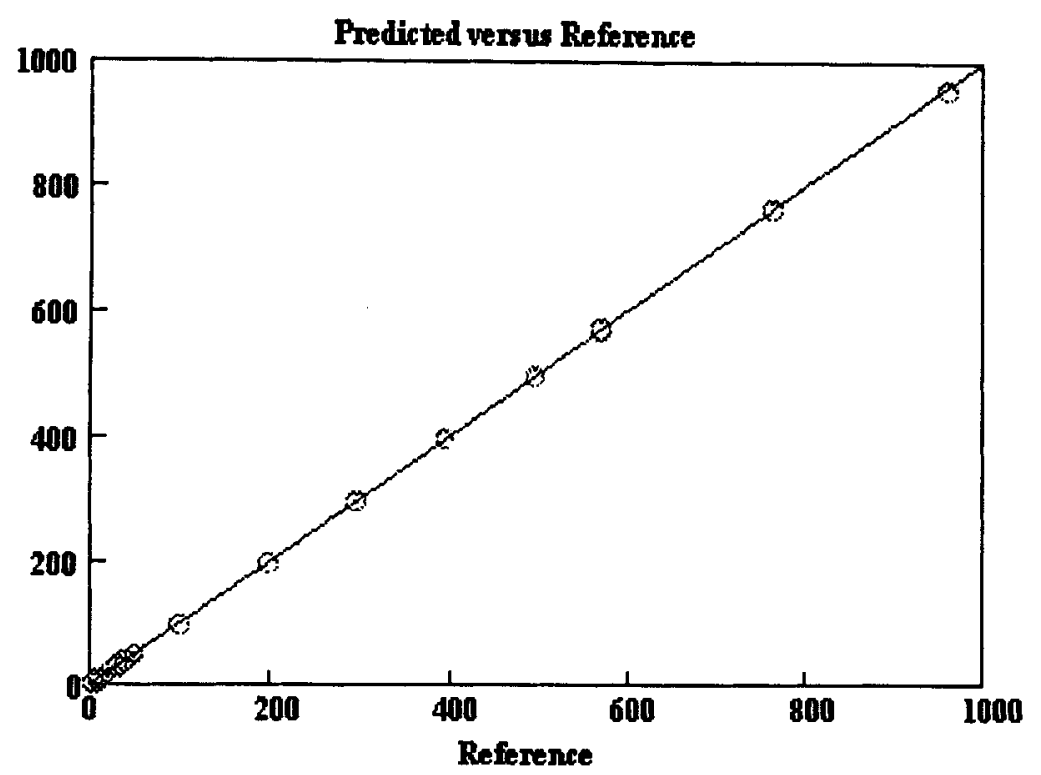

Figure 5. Predicted versus reference concentration in $\mathrm{ppm}$ for $\mathrm{SO}_{2}$ (calibration set) (CLSI).

how well the model fits each wavenumber (Fig. 8). Here it is clearly visible that the wavenumber(s) around $1360 \mathrm{~cm}^{-1}$ are very outlying compared to the other wavenumbers and it is mainly this narrow region that contributes to $F$-values which are higher than 1 . This region corresponds with the local minimum in the $\mathrm{SO}_{2}$ spectrum, which is probably difficult to account for in the CLSI model. If, for future unknowns, the residual spectrum looks like Fig. 8, then we know that it is an artefact of our model and that the prediction will therefore not be erroneous.

\section{CLSII model for $\mathrm{SO}_{2}$}

The same procedure was following to construct a CLSII model for $\mathrm{SO}_{2}$ and $\mathrm{H}_{2} \mathrm{O}$. Calibration statistics for $\mathrm{SO}_{2}$ is $\mathrm{RMSEC}=1.39 \mathrm{ppm}$, for $\mathrm{H}_{2} \mathrm{O}$ is $\mathrm{RMSEC}=533.6 \mathrm{ppm}$.

The non-linear nature of the $\mathrm{H}_{2} \mathrm{O}$ concentration-absorbance dependence is not as visible as for the results of the CLSI model in the predicted versus reference plot (Fig. 9), which proves that CLSII is more able to deal with small non-linearities as CLSI. However, the standard error of calibration for water vapour was significantly 


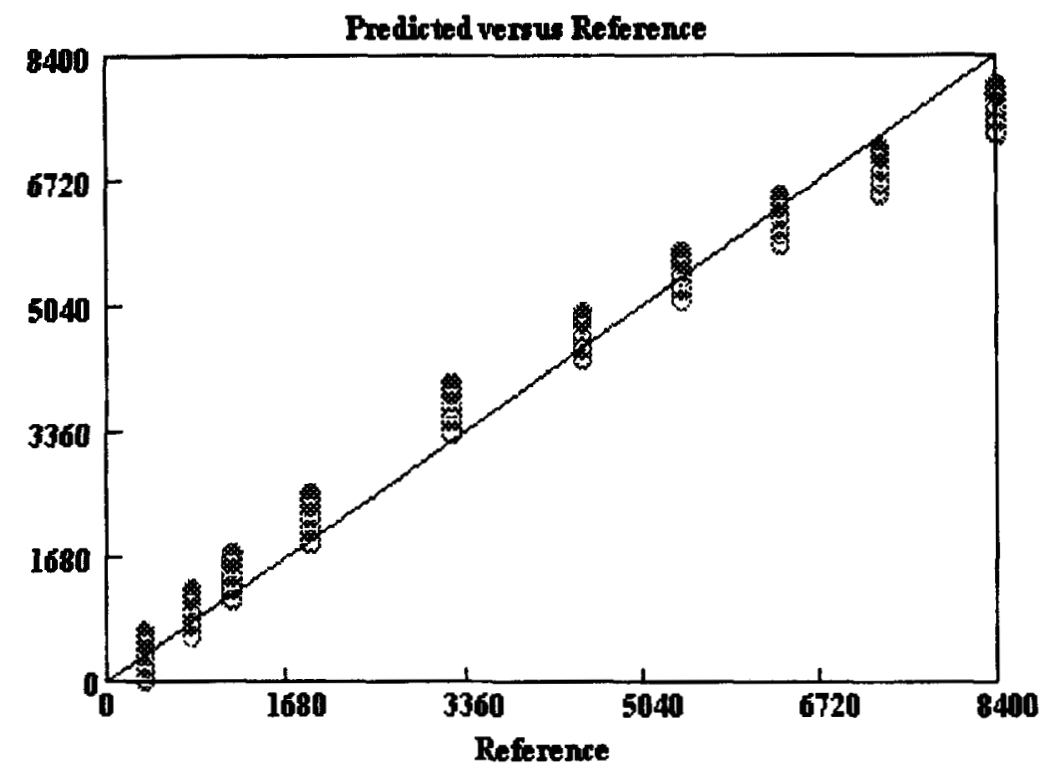

Figure 6. Predicted versus reference concentration in ppm for $\mathrm{H}_{2} \mathrm{O}$ (calibration set) (CLSI).

F-statistics : CLSI (SO2)

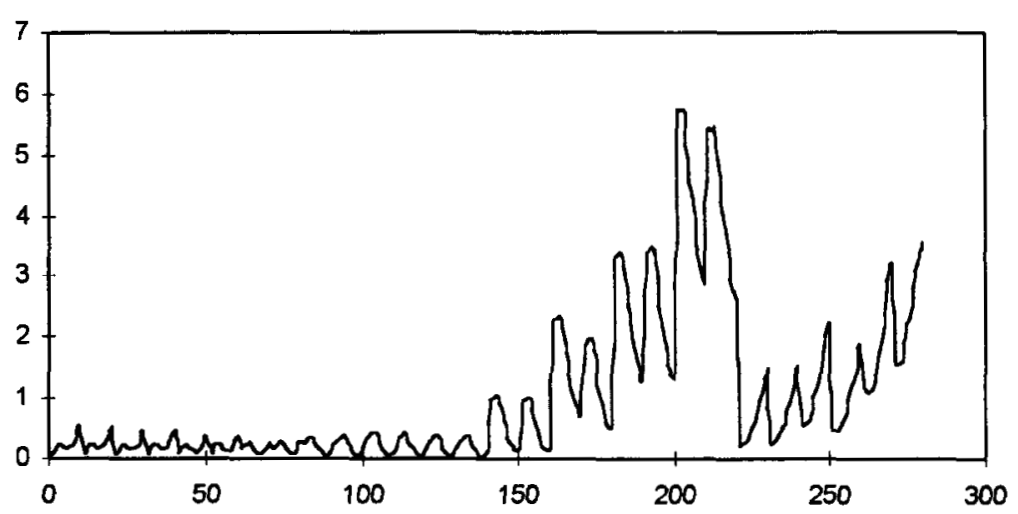

Figure 7. F-value (ordinate) calculated from the residuals (calibration diagnostics) as a function of $\mathbf{2 8 0}$ calibration samples resp. (abscissa).

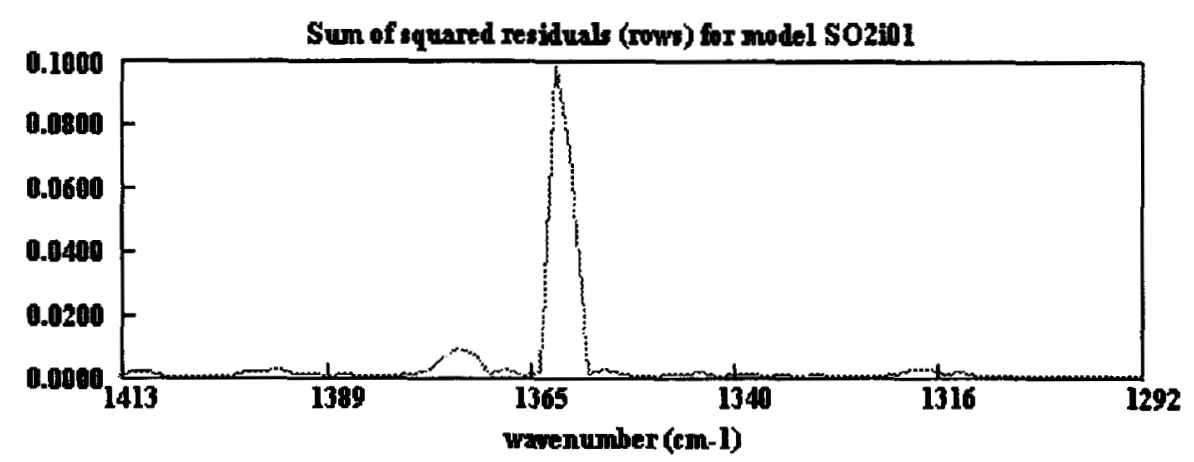

Figure 8. Sum of squares of residuals (calibration diagnostics) (arbitrary units).

higher in the CLSII case, whereas the RMSEC for $\mathrm{SO}_{2}$ was equal for both CLSI and CLSII. In both cases, CLS will be capable to give accurate predictions for $\mathrm{SO}_{2}$ and only rough estimates for $\mathrm{H}_{2} \mathrm{O}$.
The sum of squared residuals calculated over the columns were much smaller than for the CLSI model (figure not shown), although the same calibration samples again show the highest residual values or $F$-values. This indicates 


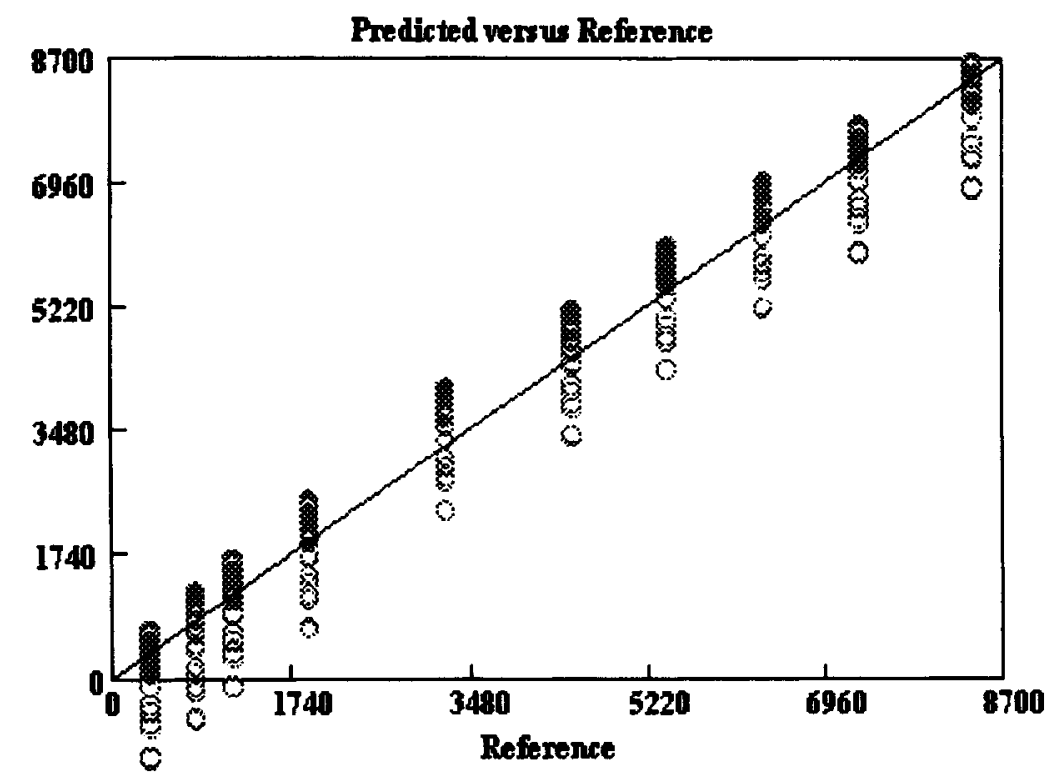

Figure 9. Predicted versus reference in ppm for $\mathrm{H}_{2} \mathrm{O}$ after CLSII calibration.

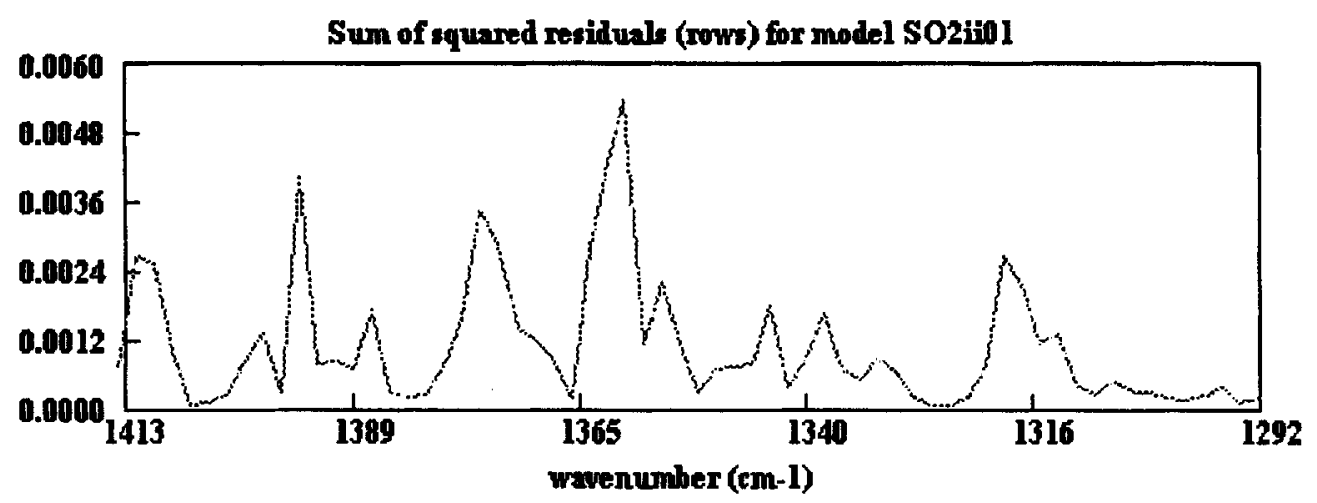

Figure 10. Sum of squares of residuals (calibration diagnostics) (arbitrary units).

that the CLSII model is better able to describe the calibration absorbance matrix A than the CLSI model.

The CLSII model seems to have fewer problems fitting the data at certain wavenumbers compared to the CLSI model (Fig. 10). This means that there are no particular parts in the selected wavenumber region that are more difficult to account for in the model. The magnitude of the spectral residuals is also much smaller for the CLSII model compared to the CLSI model (compare Fig. 8 with Fig. 10). In conclusion, by considering calibration diagnostics, the CLSII model seems to be more robust for $\mathrm{SO}_{2}$ than the CLSI model.

\section{PLS model for $\mathrm{SO}_{2}$}

The problem with PLS (PLS1 is considered) is often the choice of the number of principal components to account for in the model. A plot of the standard error of calibration (RMSEC) as a function of the number of PC's is given in Fig. 11, for the same calibration matrix composed of 280 samples and 64 wavenumbers for $\mathrm{SO}_{2}$ and $\mathrm{H}_{2} \mathrm{O}$.

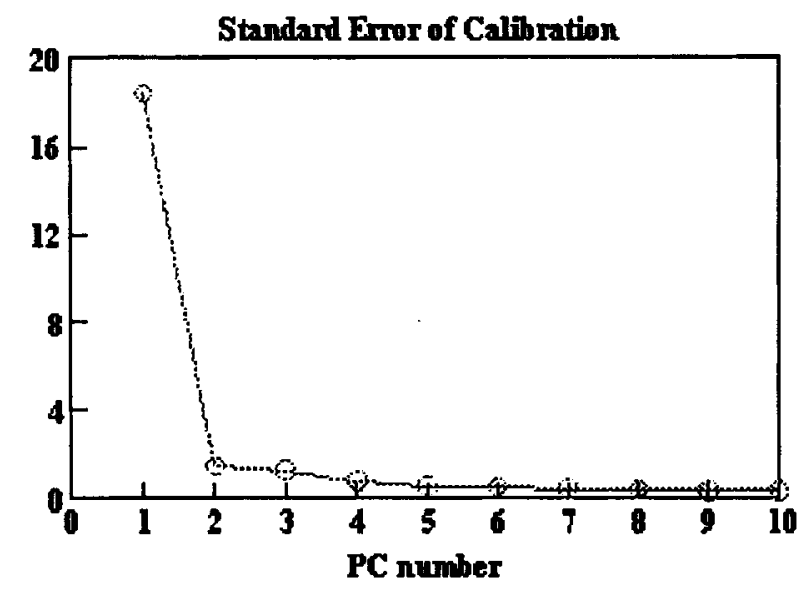

Figure 11. Standard error of calibration in ppm as a number of PCs in the PLS-model.

From the RMSEC plot (Fig. 11) it is seen that the maximum number of PCs does not need to be higher than three. Two PCs seems to be the optimal choice. PLS-models with two PCs and three PCs are considered 


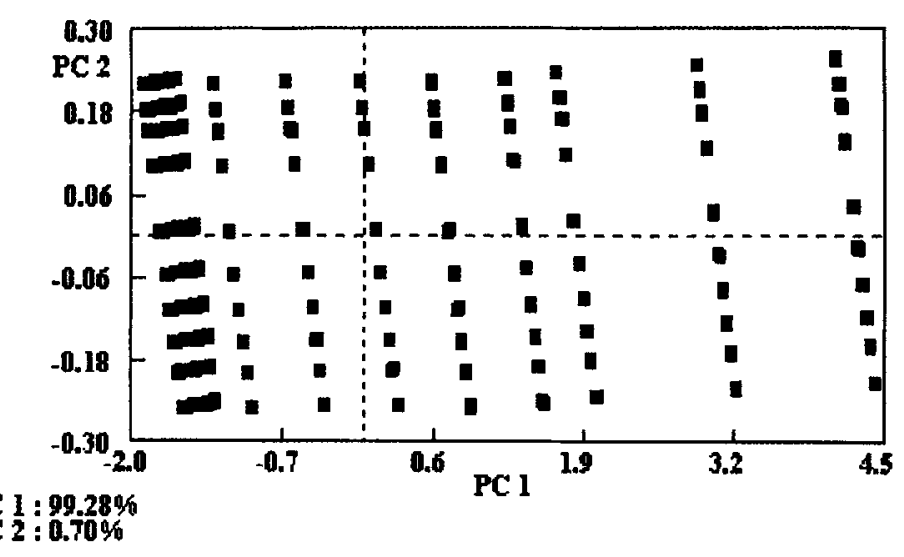

Figure 12. Two-vector scores plot for the calibration set.

in what follows in order to be able to conclude which model seems to be the most appropriate for further prediction purposes.

After PCA or PLS, one can evaluate the calibration model by considering scores and loadings in two-dimensional plots. In the case of $\mathrm{SO}_{2}$ and $\mathrm{H}_{2} \mathrm{O}$, mainly all the variance in $\mathbf{A}$ is explained by the first $\mathbf{P C}$, which means that the variables are all well correlated. However, if we consider the two-vector scores plot of the first two PCs (Fig. 12), then the points are distributed in such a way that each horizontal line explains the variance in the absorbance matrix due to the change in $\mathrm{SO}_{2}$ concentration, while each vertical line explains the variance in the absorbance matrix due to changes in the $\mathrm{H}_{2} \mathrm{O}$ concentration. The relative importance of each $\mathrm{PC}$ can also be seen from the magnitude of the coordinates of the points in the scores plot.

From the one-vector loading weights plot, it is known that if most of the variance is explained by only one PC, then the form of the loading weights as a function of the wavenumber must follow closely the real form of the $\mathrm{SO}_{2}$ spectral band. This can indeed be seen from Fig. 13.

The two-vector scores plot (Fig. 12) and one-vector loading weights plot (Fig. 13) do not depend on the number of principal components in the model. However, $B$-coefficients plotted as a function of wavenumber or predicted versus reference plots do depend on the number of PCs in the model. Two models are considered: two PCs (PLSI) and three PCs (PLSII).

\section{PLSI (two PCs): RMSEC $=1.46$ ppm}

Calibration diagnostics can be evaluated from the sum of squared residuals, calculated for each sample. Comparison of these squared residuals with the previous CLS models shows that the same samples are difficult to account for in the three models. CLSII seems to fit them the best of the three models, during calibration (Fig. 14).

Considering the squared residuals summed over the samples and plotted as a function of the wavenumber (Fig. 15), one can see that, just like the CLSI model, the PLSI model has most difficulties to fit absorbances in the

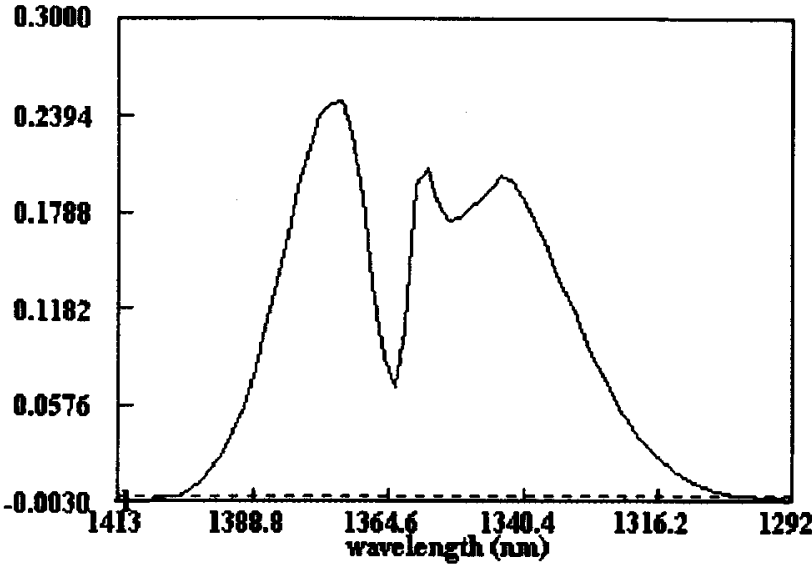

Figure 13. One-vector loading weights (arbitrary units) plot for the calibration set.

region around $1360 \mathrm{~cm}^{-1}$. This corresponds to the local minimum in the $\mathrm{SO}_{2}$ spectral band.

\section{PLSII (three PCs): RMSEC $=1.25$ ppm}

Calibration diagnostics may be considered by inspection of the sum of squared residuals plotted against sample number or wavenumber (Fig. 16). The magnitude of the squared residuals for the 3PC model is, in both cases, less than that of the squared residuals calculated from the CLSII model. However, there is a chance that we are already overfitting the data with three PCs. To check this one can use an independent test set or an internal validation technique (cross-validation) to determine the optimal number of PCs.

\section{Test set for $\mathrm{SO}_{2}$}

Calibration diagnostics is, of course, very important (for the determination of outlying calibration samples and to follow the model-building process) but the ultimate test for every model is its application to a really independent 


\section{Residuals for the SO2 calibration matrix}

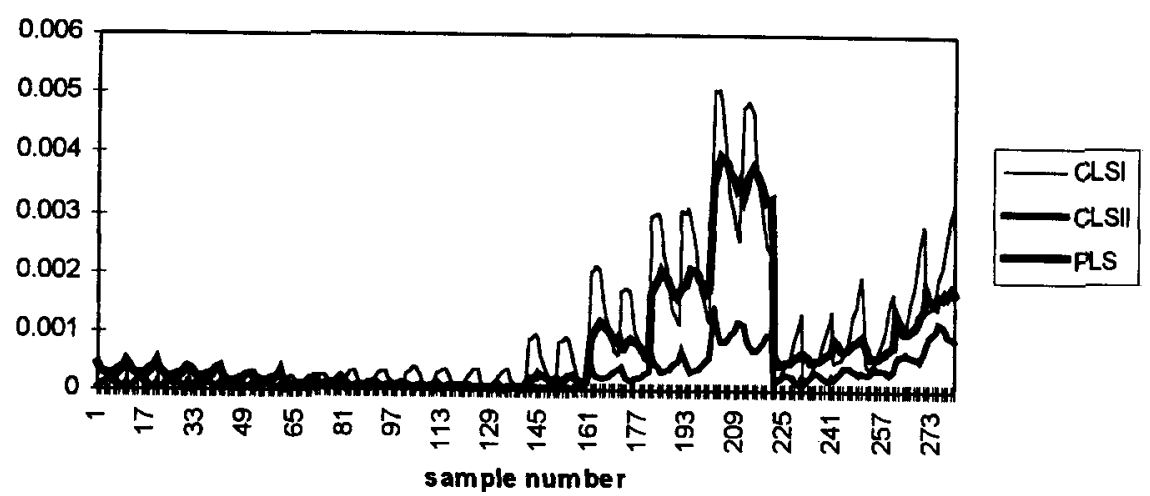

Figure 14. Comparison of sums of squared residuals for three models for $\mathrm{SO}_{2}$.

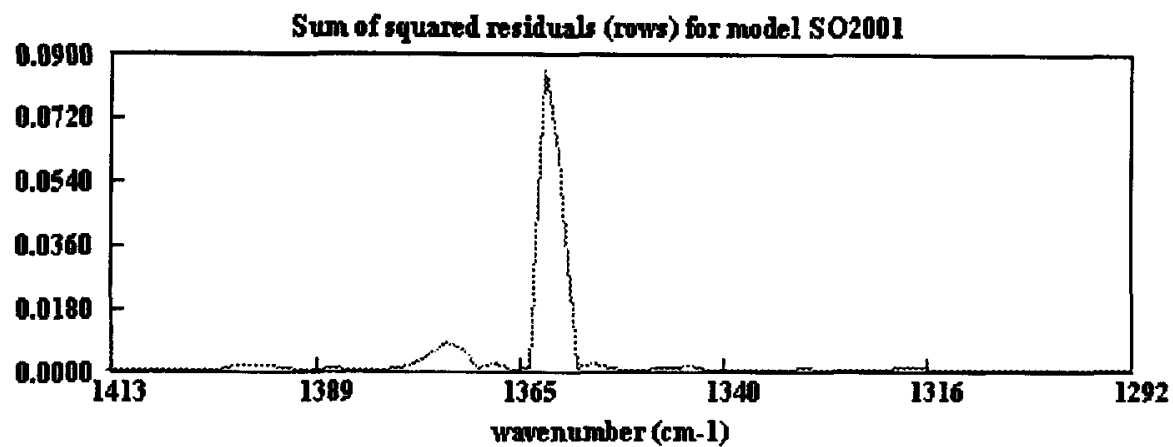

Figure 15. Sum of squared residuals (arbitrary units) (calibration diagnostics).

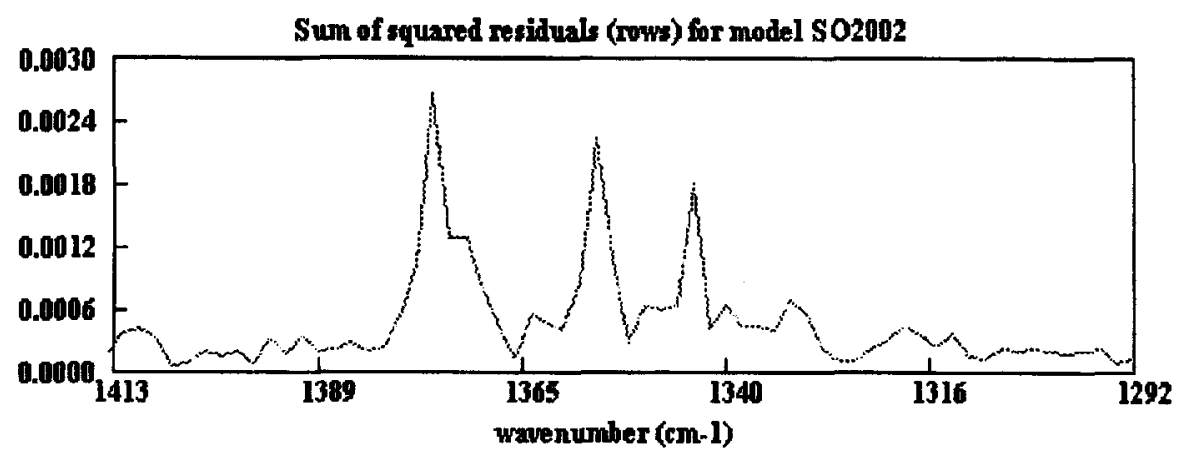

Figure 16. Sum of squared residuals (arbitrary units) (calibration diagnostics).

test set. Therefore, real mixtures of $\mathrm{SO}_{2}$ and $\mathrm{H}_{2} \mathrm{O}$ vapour (total of 60 mixtures) were made and the spectra were collected. Five models-CLSI, CLSII, PLS(2 PCs), PLS(3 PCs) and the Q-matrix method-were used to predict the $\mathrm{SO}_{2}$ concentrations. As a reference method, the water vapour spectrum is subtracted from the mixture spectrum and the $\mathrm{SO}_{2}$ concentration is determined by use of the area model (it would have been better if the $\mathrm{SO}_{2}$ concentration could be determined by one or another direct measuring technique). Prediction statistics for the $\mathrm{SO}_{2}$ test set are given in Table 1 .
All five methods have RMSEP values between $2.30 \mathrm{ppm}$ and $4.91 \mathrm{ppm}$, which means that they are all comparable to the reference method $(<5 \mathrm{ppm}$ in a range of 5-960 ppm). If the number of samples in the test set is large enough, then the RMSEP value will approach the real prediction error of the population of all future samples. Because the results are compared with another method applied on the same test set spectra, a systematic error could be introduced. This systematic error (bias) is accounted for in the SEP value, which is probably here a better parameter to judge our five models. Slope, offset 


\begin{tabular}{llllll}
\hline \multicolumn{7}{c}{ Table 1. Test set results for $\mathrm{SO}_{2}$} \\
& CLS1 & CLSII & PLS (2 PCs) & PLS (3 PCs) & Q-Matrix \\
RMSEP & 2.41 & 2.46 & 2.30 & 3.08 & 4.91 \\
Bias & 1.18 & 1.25 & 1.13 & 1.96 & 4.26 \\
SEP & 2.12 & 2.14 & 2.03 & 2.39 & 2.47 \\
Slope & 0.989 & 0.988 & 0.989 & 0.985 & 0.981 \\
Offset & 1.01 & 0.98 & 0.92 & 1.01 & -0.66 \\
Corr. & 1.000 & 1.000 & 1.000 & 1.000 & 1.000 \\
\hline
\end{tabular}

F-statistics for SO2 test set

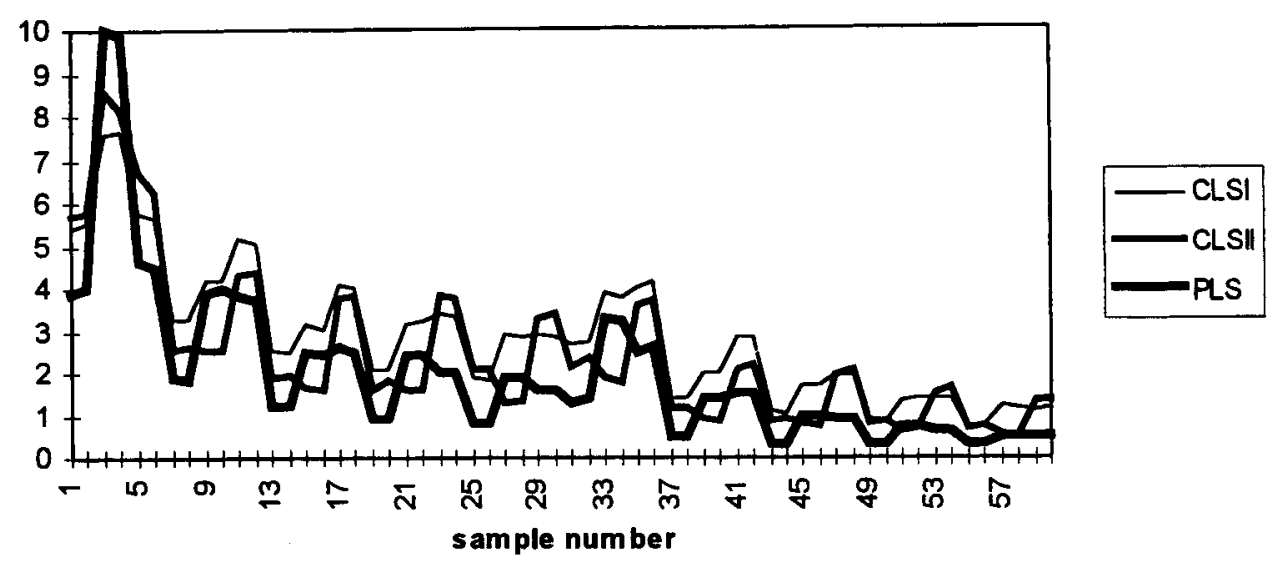

Figure 17. $\mathrm{F}$-value for the test for $\mathrm{SO}_{2}$.

and correlation coefficient of the predicted versus reference curve were also given in Table 1 . A slope $=1$, offset $=0$ and corr. $=1$ represents the ideal situation. Unexpected interferences can lead to erroneous predictions. It is therefore worthwhile to compare the sum of squared residuals of our unknown sample with the average sum of squared residuals of our calibration set. The $F$-value is defined as the ratio of these two values and, as stated earlier, has to be close to ' 1 ' if the calculated residual is of the same order of magnitude as the average residual of the calibration set. We already have seen that $F$-values, up to 6, can appear even for calibration samples without having a good reason to omit these samples. Applying $F$-statistics to our test set, we see that even $F$-values up to $10 \mathrm{can}$ be obtained. Further inspection of the residual spectrum of these samples can probably give us information about the reason for these high $F$-values. $F$-statistics for the test set for 3 models CLSI, CLSII and PLS(2 PCs) are given in Fig. 17.

A closer look to the residual spectrum for sample number 3 , for the different models CLSI, CLSII and PLS(2 PCs) can teach us something about the reason for the high $F$-value (Figs. 18-20). These residual spectra show that the major contribution to the high $F$-value for that particular sample is due to the wavenumber region around $1360 \mathrm{~cm}^{-1}$. From our calibration statistics we already knew that this region was difficult to fit by our models. Another major contribution to the $F$-value is due to fitting problems in the region around $1340 \mathrm{~cm}^{-1}$.
As a rule of thumb one can use the $F$-statistics for our $\mathrm{SO}_{2}$ models as follows:

- $F \leqslant 3$, the prediction is excellent, there will be no interference.

- $3<F \leqslant 10$, the prediction may still be good, but it is worth evaluating the form of the residual spectrum for the presence of unexpected interferences. In most cases the high $F$-value will be due to the model not being able to fit the region around $1360 \mathrm{~cm}^{-1}$ and/or $1340 \mathrm{~cm}^{-1}$.

- $F>10$, the prediction is suspicious, but can, however, still be good! This is the case when the high $F$-values are due to the model not being able to fit the water vapour absorbance part in the spectral region of interest. In this case, one has to find typical water vapour spectral bands in the residual spectrum. However, if the residual spectrum really shows a clear spectral band which may be characteristic for an unexpected interferent, the prediction will be wrong!

Another example concerns the effect of non-linearities. This can be demonstrated by applying PLS to the $\mathrm{CO}_{2} / \mathrm{H}_{2} \mathrm{O}$ interference in the $3763-3500 \mathrm{~cm}^{-1}$ region. The PLS2-model explained in reference 6 was applied to an independent test set containing 212 mixture spectra of $\mathrm{CO}_{2}$ and $\mathrm{H}_{2} \mathrm{O}$. The $\mathrm{CO}_{2}$ reference concentration was determined independently by letting the gasflow go first through the $\mathrm{CO} / \mathrm{CO}_{2}$ NDIR gas analysers of the cone calorimeter. The predicted versus reference plot for the 


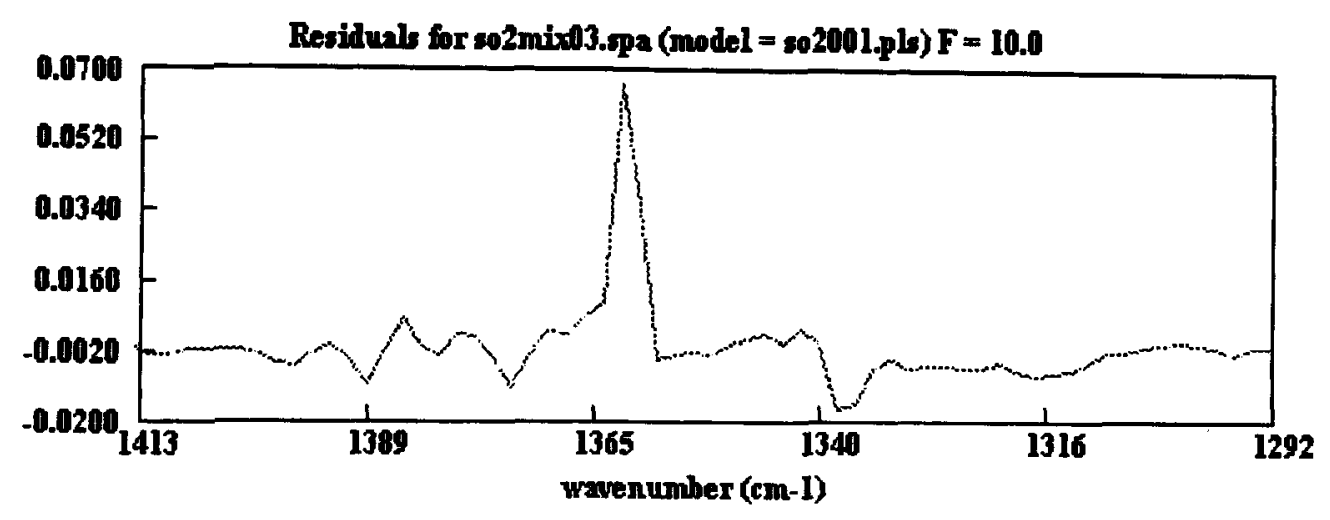

Figure 18. Residual spectrum for sample number 3 in the test set (PLSI).

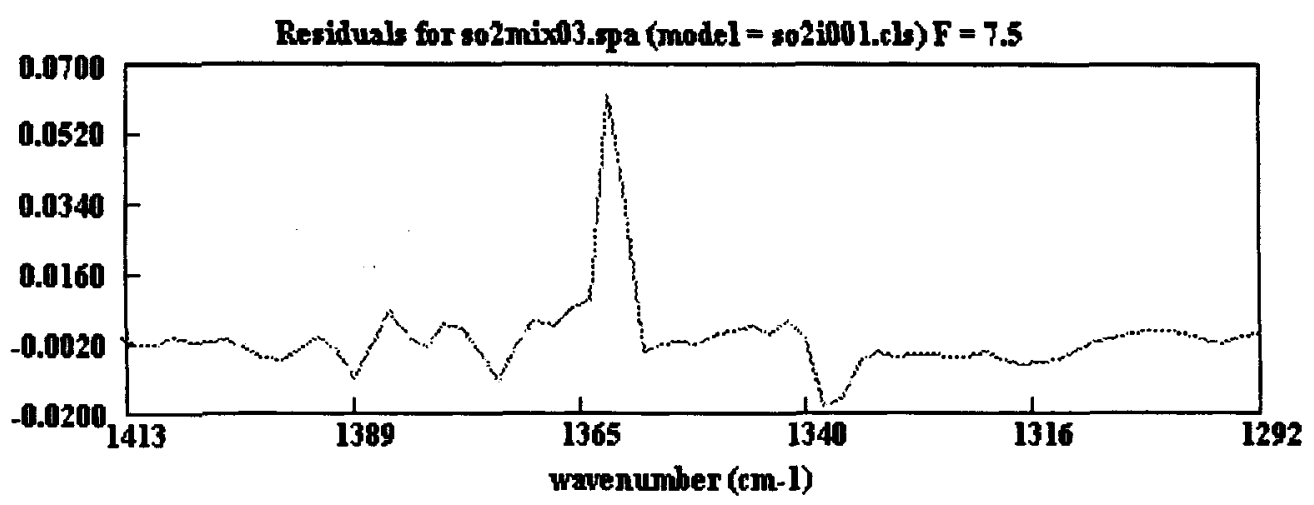

Figure 19. Residual spectrum for sample number 3 in the test set (CLSI).

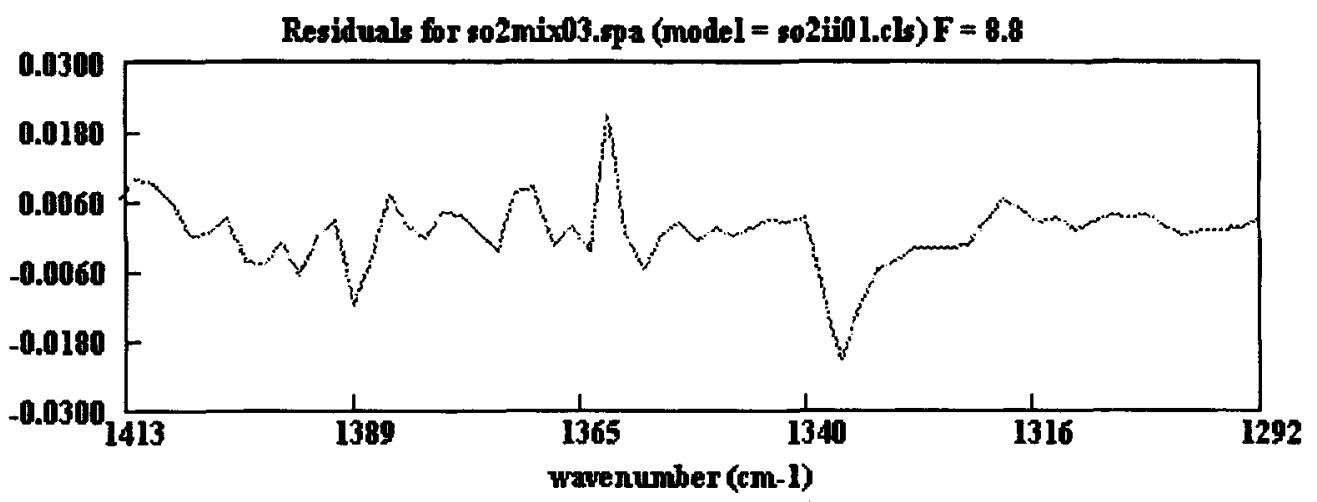

Figure 20. Residual spectrum for sample number 3 in the test set (CLSII).

212 test sample spectra for the PLS2-model is shown in Fig. 21. The non-linear behaviour is still visible in this plot, although the statistics are acceptable (Table 2).

Application of the INLR-model ${ }^{22}$ to the same test set of 212 samples resulted in a clear improvement in the test statistics, as can be seen from Table 2 . The improvement in RMSEP is more than $50 \%$, the SEP improved by nearly $75 \%$. Also, slope and bias which have to be as close as possible to 1 and 0 , are much better when quadratic terms were added to the absorbance matrix before PLS was performed. The predicted versus reference plot from Fig. 22 shows a much more linear relation.

\section{CONCLUSION}

A variety of methods, univariate and multivariate, linear and non-linear, exist to perform the quantification of gas components in real smoke gas spectra obtained with the FTIR technique. Each method has its specific advantages 


\section{PLS2 (4 PC's) on test set (212 samples)}

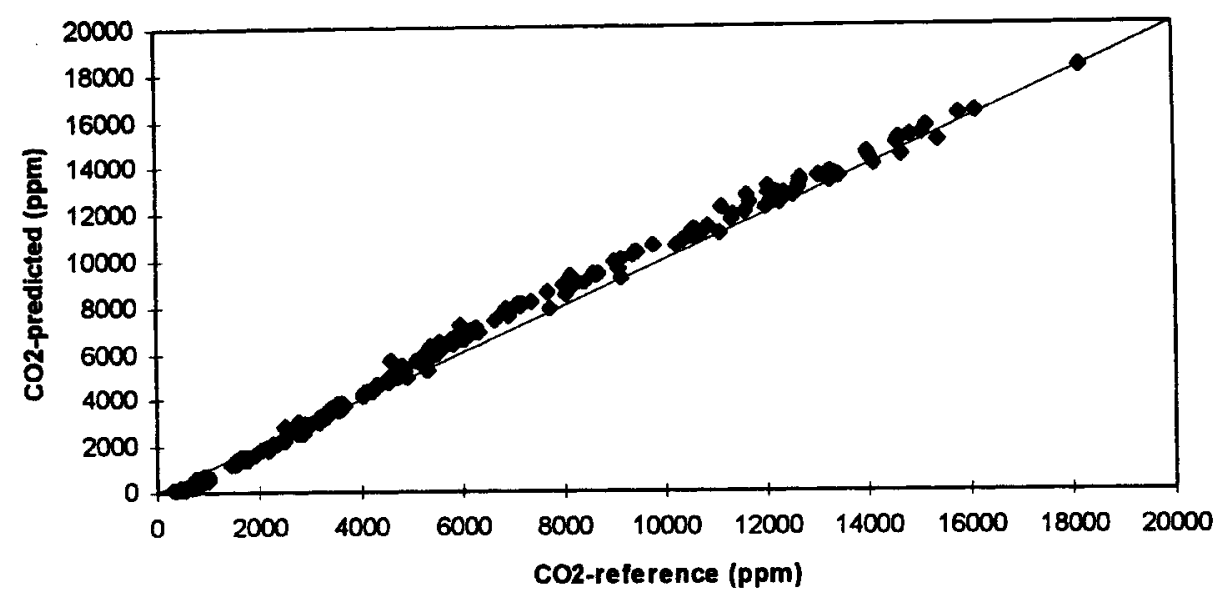

Figure 21. Predicted versus reference for the $\mathrm{CO}_{2} / \mathrm{H}_{2} \mathrm{O}$ test set (PLS2-model).

INLR (5 PC's) on test set (212 samples)

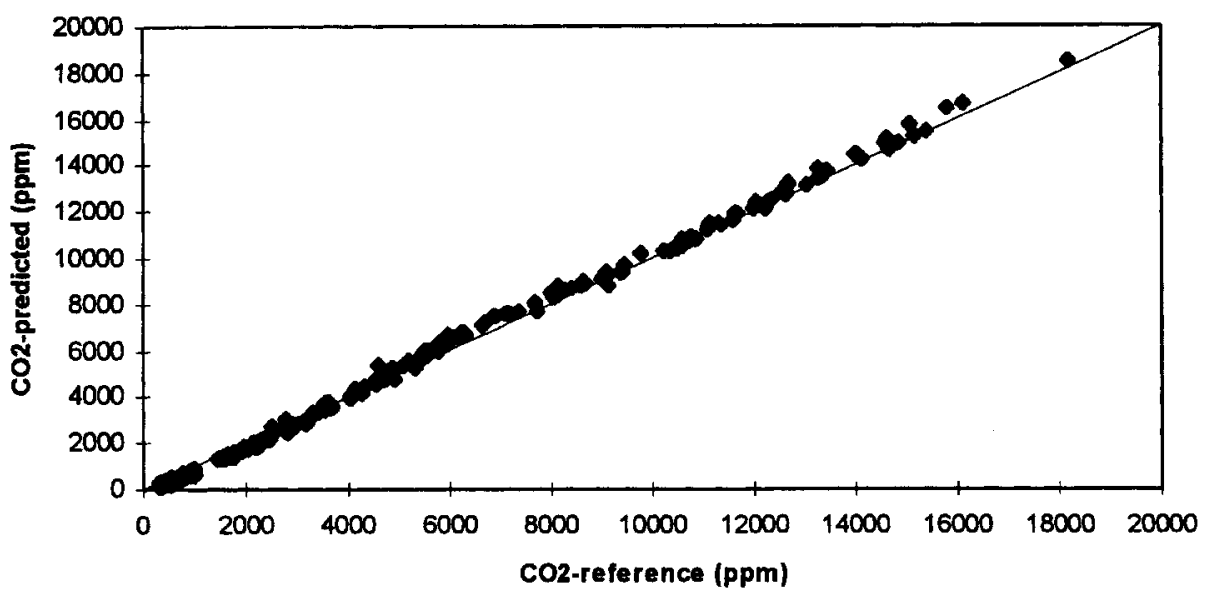

Figure 22. Predicted versus reference for the $\mathrm{CO}_{2} / \mathrm{H}_{2} \mathrm{O}$ test set (INLR model).

\begin{tabular}{lcc}
\hline Table 2. & Test set statistics for the $\mathrm{CO}_{2} / \mathrm{H}_{2} \mathrm{O}$ data set \\
& PLS2 & 236 \\
RMSEP & 504 & 62 \\
Bias & -255 & 158 \\
SEP & 436 & 1.018 \\
Slope & 1.055 & -125 \\
Offset & -86 & 0.999 \\
Corr. & 0.997 & \\
\hline
\end{tabular}

and disadvantages and each of these have to be weighted against each other before the most appropriate choice can be made for each individual gas component. It has been shown that in the case of overlapping spectral features, full-spectrum methods have to be preferred and that for gas components such as $\mathrm{SO}_{2}$, and also for $\mathrm{C}_{3} \mathrm{H}_{4} \mathrm{O}$, which show a linear absorbance-concentration dependence at a bandwidth resolution of $4 \mathrm{~cm}^{-1}$, most of the linear full-spectrum methods give acceptable accuracy. In contrast, for gas molecules as $\mathrm{CO}_{2}$, and also for $\mathrm{CO}$ and others such as $\mathrm{HCl}, \mathrm{HBr}, \mathrm{HCN}, \mathrm{NO}, \mathrm{NO}_{2}, \ldots$, non-linear models such as INLR will give improved results compared to the linear full-spectrum methods. Another improvement might be splitting up the region of interest in separate regions to avoid the most strongly overlapping spectral bands, e.g. in the case of $\mathrm{SO}_{2}$, the region around $1360 \mathrm{~cm}^{-1}$ may be omitted, which would probably improve the diagnostics based on $F$-statistics. These statistics or diagnostic procedures based on residual spectra are another important advantage of fullspectrum methods over methods where only a few wavenumbers are used in the analysis. 
1. M. R. Nyden and V. Babrauskas, Use of FTIR spectroscopy for multicomponent quantitation in combustion toxicology. Combined Technical Meetings: Eastern Section, the Combustion Institute and the Center for Flre Research Annual Conference on Fire Research, Gaithersburg MD, 1987, 107, $1-4$.

2. R. Kallonen, Smoke gas analysis by FTIR Method. Preliminary investigation. Journal of Fire Sciences 8, 343-360 (1990).

3. R. Kallonen and E. Mikkola, Smoke gas analysis by FTIR (Nordtest-project No. 970-91).

4. J. Bak and A. Larsen, Quantitative gas analysis with FTIR: a method for $\mathrm{CO}$ calibration using partial least squares with linearized data. Applied Spectroscopy 49, 4, 437-443 (1995).

5. J. Tetteh, E. Metcalfe, S. Howells, R. Withnall, S. Ames and S. Vollam, Orthogonal noise annihilation for FTIR spectroscopy in fire gas analysis. Fire and Materials 20, 51-59 (1996).

6. H. Pottel, The use of partial least squares (PLS) in quantitative FTIR-determination of gas concentrations in smoke gases of burning textiles. Fire and Materials 19, 221-231 (1995).

7. C.W. Brown and R. J. Obremski, Multicomponent quantitative analysis. Applied Spectroscopy Reviews 20, 3, 373-418 (1984).

8. D. M. Haaland and R. G. Easterling, Application of new leastsquares methods for quantitative infrared analysis of multicomponent samples. Applied Spectroscopy 36, 6, 665-673 (1982).

9. D. M. Haaland, R. G. Easterling and D. A. Vopicka, Multivariate least squares method applied to the quantitative spectral analysis of multicomponent samples. Applied Spectroscopy 39, 1, 73-84 (1985).

10. S. C. Rutan, Kalman filtering approaches for solving problems in analytical chemistry. Journal of Chemometrics 1, 7-18 (1987).

11. S. D. Brown, Rapid parameter estimation with incomplete chemical calibration models. Chemometrics and Intelligent Laboratory Systems 10, 87-105 (1991).

12. L. V. Pérez-Arribas, F. Navarro-Villoslada, M. E. Leon-Gonzalez and L. M. Polo-Diez, Use of the Kalman filter for multivariate calibration in a real system and its comparison with CLS and pure component calibration methods. Journal of Chemometrics 7, 267-275 (1993).

13. C. L. Erickson, M. J. Lysaght and J. B. Callis, Relationship between digital filtering and multivariate regression in quantitative analysis. Analytical Chemistry 64, 24, 1155-1163 (1992).

14. D. M. Haaland and E. V. Thomas, Partial least squares methods for spectral analyses. 1. Relation to other quantitat- ive calibration methods and the extraction of qualitative information. Analytical Chemistry 60, 11, 1193-1202 (1988).

15. G. L. McClure, P. B. Roush, J. F. Williams and C. A. Lehmann, Application of computerized quantitative infrared spectroscopy to the determination of the principal lipids found in blood serum. Computerized Quantitative Infrared Analysis 131-155 (1987).

16. S. Wold, K. Esbensen and P. Geladi, Principal component analysis. Chemometrics and intelligent Laboratory Systems 2, 37-52 (1987).

17. H. Martens and T. Naes, Multivariate Calibration, Wiley, Chichester (1989).

18. E. R. Malinowski and D. G. Howery, Factor Analysis in Chemistry, Wiley, New York (1980)

19. P. M. Fredericks, J. B. Lee, P. R. Osborn and D. A. J. Swinkels, Materials characterization using factor analysis of FTIR spectra. Part 1: Results. Applied Spectroscopy 39, 2, 303-310 (1985).

20. P. M. Fredericks, J. B. Lee, P. R. Osborn and D. A. J. Swinkels, Materials characterization using factor analysis of FTIR spectra. Part 2: Mathematical and statistical considerations. Applied Spectroscopy 39, 2, 311-316 (1985).

21. P. Geladi and B. R. Kowalski, Partial Least Squares Regression: a tutorial. Analytica Chimica Acta 185, 1-17 (1986).

22. A. Berglund and S. Wold, INLR, Implicit Non Linear Latent variable Regression. 4th Scandinavian Symposium of Chemometrics (1995).

23. B. R. Kowaiski and M. B. Seasholtz, Recent developments in multivariate calibration. Journal of Chemometrics 5 , 129-145 (1991).

24. I. E. Frank, A nonlinear PLS model. Chemometrics and Intelligent Laboratory Systems 8, 109-119 (1990).

25. S. Wold, N. Kettaneh-Wold and B. Skagerberg, Nonlinear PLS modelling. Chemometrics and Intelligent Laboratory Systems 7, 53-65 (1989).

26. J. Zupan and J. Gasteiger, Neural Networks for Chemists, An Introduction, $\mathrm{VCH}$ Verslagsgesellschaft $\mathrm{mbH}$, Weinheim (1993).

27. Y. H. Pao, Adaptive Pattern Recognition and Neural Networks, Addison Wesley, Reading, MA (1989).

28. Y. Liu, B. R. Upadhyaya, M. Naghedolfeizi, Chemometric data analysis using artificial neural networks. Applied Spectroscopy 47, 1, 12-23 (1993).

29. W. J. Jasper, E. T. Kovacs and G. Berkstresser, Using neural networks to predict dye concentrations in multiple-dye mix tures. Text. Res. J. 63, 545-550 (1993).

30. T. Naes, K. Kvaal, T. Isaksson and C. Miller, Artificial neural networks in multivariate calibration. Journal of Near Infrared Spectroscopy 1, 1-11 (1993). 